\title{
A variational analysis of the spinorial Yamabe equation on product manifolds
}

\author{
Yannick Sire, Tian $\mathrm{Xu}^{*}$
}

\begin{abstract}
This work is devoted to the analysis of the Yamabe problem on Spin manifolds and some applications to CMC immersions. Despite the efforts of many authors, very little is known on the existence of Yamabe metrics on general Spin manifolds. Motivated to bubbling phenomena for the Riemannian problem and recent multiplicity results in this setting, we investigate special spinorial Yamabe metrics on product manifolds developing a bubbling analysis which has independent interest in the present setting.
\end{abstract}

MSC 2010: Primary: 53C27; Secondary: 35R01

Keywords. Spin structure, product manifolds, Spinorial Yamabe problem, bubbling analysis

\section{Contents}

1 Introduction and setting of the problem

2 The setting 5

2.1 Some algebraic preliminaries . . . . . . . . . . . . . . 5

2.2 The Dirac operator . . . . . . . . . . . . . . . . . . 7

2.3 Analysis on a product conformal structure . . . . . . . . . . 8

2.4 Functional framework . . . . . . . . . . . . . . . . . 9

3 Existence of solutions

4 Energy gap for solutions in Euclidean spaces: the bubbles 18

5 Bubbling analysis $\quad 20$

6 Asymptotic profiles of the solutions 24

7 Application: CMC immersions for Unduloids 29

${ }^{*}$ Supported by the National Science Foundation of China (NSFC 11601370) and the Alexander von Humboldt Foundation of Germany 


\section{Introduction and setting of the problem}

The well known Yamabe problem seeks for the existence of a constant scalar curvature metric in a given conformal class of Riemannian metrics on a compact manifold. Such a metric can be characterized variationally as a critical point of the Hilbert-Einstein functional on conformal classes. A positive answer to this problem, obtained in a series of steps by H. Yamabe [45], T. Aubin [12], N. Trudinger [44] and R. Schoen [41], provides at least one minimizer of the Hilbert-Einstein functional in each conformal class. Such a metric is called a Yamabe metric. To determine all the Yamabe metrics in a given conformal class is generally a very difficult problem particularly when the scalar curvature has positive sign. It is interesting to observe that, generically, minima of the Hilbert-Einstein functional in conformal classes are unique, see [11]. However, in many cases a rich variety of constant scalar curvature metrics arise as critical points that are not necessarily minimizers, and it is a very interesting task to classify those metrics. Multiplicity of solutions of the Yamabe problem has been studied in the literature, especially in product manifolds, several results have been obtained in the special case of products with round spheres, see for instance [26, 31, 39, 42] and references therein.

In the setting of Spin Geometry, a problem analogous to the Yamabe problem has received increasing attention in recent years. Several works of Ammann [?, 4, 6] and Ammann, Humbert et al [8-10] provide a framework in which variational methods may be employed.

Let $(M, g, \sigma)$ be an $m$-dimensional closed spin manifold with a metric $g$, a spin structure $\sigma$ : $P_{S p i n}(M) \rightarrow P_{S O}(M)$. With the notation $\rho: \operatorname{Spin}(m) \rightarrow \operatorname{End}\left(\mathbb{S}_{m}\right)$ be the spin representation, we denote $\mathbb{S}(M)=P_{S p i n}(M) \times{ }_{\rho} \mathbb{S}_{m}$ the spinor bundle over $M$ and $D_{g}^{M}: C^{\infty}(M, \mathbb{S}(M)) \rightarrow$ $C^{\infty}(M, \mathbb{S}(M))$ the Dirac operator (see [24, 34] for more geometric backgrounds). Analogous to the Yamabe invariant, a spin conformal invariant is defined as

$$
\lambda_{m i n}^{+}(M,[g], \sigma):=\inf _{\tilde{g} \in[g]} \lambda_{1}^{+}(\tilde{g}) \operatorname{Vol}(M, \tilde{g})^{\frac{1}{m}}
$$

where $\lambda_{1}^{+}(\tilde{g})$ denotes the smallest positive eigenvalue of the Dirac operator $D_{\tilde{g}}^{M}$ with respect to the conformal metric $\tilde{g} \in[g]:=\left\{f^{2} g: f \in C^{\infty}(M), f>0\right\}$. Ammann points out in [4,6] that studying critical metrics for this invariant involves similar analytic problems to those appearing in the Yamabe problem. It follows that finding a critical metric of (1.1) is equivalent to prove the existence of a spinor field $\psi \in C^{\infty}(M, \mathbb{S}(M))$ minimizing the functional defined by

$$
J_{g}(\phi)=\frac{\left(\int_{M}\left|D_{g}^{M} \phi\right|^{\frac{2 m}{m+1}} d \operatorname{vol}_{g}\right)^{\frac{m+1}{m}}}{\left|\int_{M}\left(D_{g}^{M} \phi, \phi\right) d \operatorname{vol}_{g}\right|}
$$

with the Euler-Lagrange equation

$$
D_{g}^{M} \psi=\lambda_{\min }^{+}(M,[g], \sigma)|\psi|_{g}^{2^{*}-2} \psi
$$

where $m^{*}:=\frac{2 m}{m-1}$.

As was pointed out in [4], standard variational method does not imply the existence of minimizers for $J_{g}$ directly. This is due to the criticality of the nonlinearity in (1.3). Indeed, the exponent $m^{*}=\frac{2 m}{m-1}$ is critical for the corresponding Sobolev embedding. Similar to the argument in solving the Yamabe problem, one might be able to find a criterion which recovers the 
compactness. It is crucial to note that a spinorial analogue of Aubin's inequality holds (see [8])

$$
\lambda_{\text {min }}^{+}(M,[g], \sigma) \leq \lambda_{\text {min }}^{+}\left(S^{m},\left[g_{S^{m}}\right], \sigma_{S^{m}}\right)=\frac{m}{2} \omega_{m}^{\frac{1}{m}}
$$

where $\left(S^{m}, g_{S^{m}}, \sigma_{S^{m}}\right)$ is the $m$-dimensional sphere equipped with its canonical metric $g_{S^{m}}$ and its standard spin structure $\sigma_{S^{m}}$, and $\omega_{m}$ is the standard volume of $\left(S^{m}, g_{S^{m}}\right)$. The criterion obtained in [4] shows that if inequality (1.4) is strict then the spinorial Yamabe problem (1.3) has a nontrivial solution minimizing the functional $J_{g}$. However, the strict inequality in (1.4) is only verified for some special cases and general results are still missing (cf. [7, 10, 25]).

Tightly related to geometric data, the nonlinear problem (1.3) provides a strong tool for showing the existence of constant mean curvature hypersurfaces in Euclidean spaces. This has been known as the Spinorial Weierstraß representation, see for instance [4,23,33]. This is one of the most attractive features of the spinorial Yamabe problem that unseals new researches in both PDE theory and Riemannian geometry. Not being confined by the strict inequality in (1.4), the purpose of this paper is to establish some existence of multiple solutions of (1.3) on products of compact spin manifolds which are not necessarily minimizers of the functional defined in (1.2).

Let us describe our results more precisely. Given closed spin manifolds $\left(M_{1}, g^{(1)}, \sigma_{1}\right)$ and $\left(M_{2}, g^{(2)}, \sigma_{2}\right)$, with fixed spin structures, we consider a family of metrics $g_{\ell}$ on the product $N=M_{1} \times M_{2}$ defined by $g_{\ell}=\ell^{2} g^{(1)} \oplus g^{(2)}, \ell>0$. Noting $m_{1}$ and $m_{2}$ the dimensions of $M_{1}$ and $M_{2}$ respectively, we will be interested in solutions of the (normalized) spinorial Yamabe equation on the product manifold $\left(N, g_{\ell}\right)$ :

$$
D_{g_{\ell}}^{N} \phi=|\phi|_{g_{\ell}}^{\frac{2}{n-1}} \phi
$$

where $n:=\operatorname{dim} N=m_{1}+m_{2}$. For simplicity we first describe here the case $n$ is odd, but the statement is similar for $n$ even, see Theorem 6.5. Without loss of generality, we may assume $m_{1}$ is even (otherwise, it is equivalent to consider the product manifold equipped with the metrics $\left.g^{(1)} \oplus \ell^{2} g^{(2)}\right)$. In this setting, the spinor bundle over $N$ can be identified with $\mathbb{S}(N)=\mathbb{S}\left(M_{1}\right) \otimes$ $\mathbb{S}\left(M_{2}\right)$ and the Dirac operator is given by

$$
D_{g_{\ell}}^{N}(\psi \otimes \varphi)=D_{\ell^{2} g^{(1)}}^{M_{1}} \psi \otimes \varphi+\omega_{\mathbb{C}}^{M_{1}} \cdot \ell^{2} g^{(1)} \psi \otimes D_{g^{(2)}}^{M_{2}} \varphi
$$

for $\psi \in C^{\infty}\left(M_{1}, \mathbb{S}\left(M_{1}\right)\right)$ and $\varphi \in C^{\infty}\left(M_{2}, \mathbb{S}\left(M_{2}\right)\right)$, where $D_{\ell^{2} g^{(1)}}^{M_{1}}$ and $D_{g^{(2)}}^{M_{2}}$ denote the Dirac operators on $M_{1}$ and $M_{2}$ respectively, $\omega_{\mathbb{C}}^{M_{1}}$ is the chirality operator in the Clifford bundle over $M_{1}$ and " $\ell_{\ell^{2} g^{(1)}}$ " is the representation of Clifford multiplication on the spinor bundle $\mathbb{S}\left(M_{1}\right)$ with respect to the metric $\ell^{2} g^{(1)}$ (see Section $\_$for detailed definitions of these notations). One can use a specials ansatz for the solutions $\phi=\psi \otimes \varphi_{\lambda}, \lambda>0$, with $\varphi_{\lambda}$ being an eigenspinor of the second factor, i.e. $D_{g^{(2)}}^{M_{2}} \varphi_{\lambda}=\lambda \varphi_{\lambda}$. A further assumption on such an ansatz is that the component $\varphi_{\lambda}$ is normalizable in the sense one can normalize $\varphi_{\lambda}$ so that $\left|\varphi_{\lambda}\right|_{g^{(2)}} \equiv 1$ almost everywhere with respect to the canonical measure on $\left(M_{2}, g^{(2)}\right)$. Then $\phi=\psi \otimes \varphi_{\lambda}$ solves the Yamabe equation (1.5) if and only if $\psi$ solves

$$
D_{\ell^{2} g^{(1)}}^{M_{1}} \psi+\lambda \omega_{\mathbb{C}}^{M_{1}} \cdot \ell^{2} g^{(1)} \psi=|\psi|_{\ell^{2} g^{(1)}}^{\frac{2}{m-1}} \psi \quad \text { on } M_{1} .
$$

One of the motivations of the present work comes from the geometric bifurcation theory in order to prove multiplicity and qualitative behaviour of Riemannian Yamabe metrics. This problem goes back to the seminal work of Schoen [42] and has been deeply investigated in several 
works (see e.g. [17.-19] and references therein). Together with Bettiol and Piccione [20], the first author investigated the multiplicity of constant $Q$-curvature metrics in a similar product manifold as in our setting but in the framework of Berger spheres. In there, the authors considered Gromov-Hausdorff limits of Einstein Riemannian minimal submersions under a parameter $\ell$. The method used in geometric bifurcation theory is the use of a general bifurcation result that detects bifurcations under a jump of the Morse index of the Jacobi operator. From this point of view, our problem is substantially more difficult than the Riemannian case since the functional is strongly indefinite, i.e. the linearization has an infinite number of eigenvalues and such a criterion for bifurcating solutions cannot be used. Another issue in the spinorial setting is that there is no significant difference in showing one solution and multiple solutions, unless one can find a way to distinguish these solutions (not simply by energies). The method developed here allows actually to distinguish the solutions.

We will find solutions to the spinorial Yamabe equation (1.5) by solving (1.6). The point here is that the problem becomes subcritical and the existence of solutions is easy to prove by variational techniques. Our strategy is the following:

- We first construct for an infinite number of values of the parameter $\ell$ a solution to (1.6).

- Then, we investigate the bubbling phenomenon as $\ell \rightarrow \infty$.

- Coming back to the original problem (1.5), a quantization formula allows to distinguish each of these solutions.

We now state our main results. The first one is the description in dimension greater or equal to 3 of the infinite family of solutions of the spinorial Yamabe problem under consideration (see Theorem 6.5 for a more precise statement) .

Theorem 1.1. There exists $\ell_{0}>0$ (possibly depending on $\lambda$ ) such that for any $\ell>\ell_{0}$ there is a non-trivial solution $\psi_{\ell}$ of (1.6) which is highly concentrated in the sense that, as $\ell \rightarrow \infty$, there exists a converging sequence $\xi_{\ell} \rightarrow \xi_{0} \in M_{1}$ such that $\left|\psi_{\ell}\left(\xi_{\ell}\right)\right| \rightarrow+\infty$ and $\left|\psi_{\ell}\right| \rightarrow 0$ uniformly on compact subsets of $M_{1} \backslash\left\{\xi_{0}\right\}$.

Furthermore, the spinor field $\phi_{\ell, \lambda}:=\psi_{\ell} \otimes \varphi_{\lambda}$ defines a generalized conformal metric $g_{\ell, \lambda}=$ $\left|\phi_{\ell, \lambda}\right|_{g_{\ell}}^{\frac{4}{n-1}} g_{\ell}$ on $N=M_{1} \times M_{2}$ (in the sense of Ammann [6. Section 3]) such that

$$
\lim _{\ell \rightarrow \infty} \frac{\operatorname{Vol}\left(N, g_{\ell, \lambda}\right)}{\lambda^{m_{2}}}=C_{M_{1}, M_{2}}
$$

where $C_{M_{1}, M_{2}}>0$ is a constant depending only on $\left(M_{1}, g^{(1)}\right)$ and $\left(M_{2}, g^{(2)}\right)$.

An interesting corollary of the above statements is for the case $N=M_{1} \times S^{1}$. Since all eigenspinors on $S^{1}$ are normalizable, substitute different eigenvalues of $\lambda$ in (1.6), one obtains multiple solutions for large $\ell$ as the volume functional $\operatorname{Vol}\left(N, g_{\ell, \lambda}\right)$ can be distinguished by varying the values of $\lambda$.

The case $m=2$ is of particular interests because we are concerned with the 2-dimensional torus, i.e. $N=S^{1} \times S^{1}$ equipped with the family of metrics $g_{\ell}:=\ell^{2} d^{2} t \oplus d^{2} \tau$ with $(t, \tau) \in$ $[0,2 \pi] \times[0,2 \pi]$ being the standard parameterizations.

Our second theorem is an application of the previous analysis. We refer the reader to Theorem 7.3 for a precise statement. 
Theorem 1.2. There exists a non-trivial solution of constant length $\lambda$ of (1.6) for all $\ell>0$.

Furthermore, at all value $\ell^{*} \in\left\{\frac{1}{2 \lambda}, \frac{2}{2 \lambda}, \ldots, \frac{n}{2 \lambda}, \ldots\right\}$, there is a bifurcating branch of solutions issuing from the constant length solution branch, and these branches consist of periodic solutions that do not have the same fundamental period;

Finally if $N=S^{1} \times S^{1}$ is equipped with the so-called non-trivial spin structure $\sigma_{N}^{*}$ then the strict inequality in (1.4) is valid, i.e.

$$
\lambda_{\min }^{+}\left(N, g_{\ell}, \sigma_{N}^{*}\right)<2 \sqrt{\pi}
$$

for all $\ell>0$.

The assumption that there exists normalizable eigenspinor on $M_{2}$ is rather harmless. This is satisfied by a large class of manifolds including the circle, the spheres and any spin $m$-manifold which can be immersed into $\mathbb{R}^{m+1}$ with constant mean curvature (see [4, Chapter 5] and [7, 10]).

\section{The setting}

\subsection{Some algebraic preliminaries}

Our aim is to derive the Dirac operator on Riemannian products of spin manifolds. In particular, we have to compare the spinor bundle of the ambient space with the spinor bundles of the factor manifolds. The starting point is the splitting of the tangent bundle of the large manifold into direct sum of two vector bundles associated with the two factors. Instructional materials can be found in [34, Chapter I. 5 and II. 7], but here we want to make it more explicit.

Let us denote by $\left\{e_{1}, \ldots, e_{m}\right\}$ the canonical basis of an oriented Euclidean space $V$ and by $\mathbb{C} \ell(V)$ the complex Clifford algebra of $V$ with its multiplication being denoted by ".". In case the dimension $m$ of $V$ is even, i.e. $m=2 k$, the Clifford algebra is isomorphic to the algebra $\mathcal{M}\left(2^{k} ; \mathbb{C}\right)$ of all complex matrices of rank $2^{k}$. Hence $\mathbb{C} \ell(V)$ has precisely one irreducible module, the spinor module $\mathbb{S}_{2 k}$ with $\operatorname{dim} \mathbb{S}_{2 k}=2^{k}$. For ease of notations, we simply write the Clifford representation as

$$
\mathbb{C} \ell(V) \otimes \mathbb{S}_{2 k} \rightarrow \mathbb{S}_{2 k}, \quad \xi \otimes \psi \mapsto \xi \cdot \psi
$$

When restricted this representation to the even subalgebra $\mathbb{C} \ell^{0}(V)$, the module $\mathbb{S}_{2 k}$ splits into two irreducible unitary representations $\mathbb{S}_{2 k}=\mathbb{S}_{2 k}^{+} \oplus \mathbb{S}_{2 k}^{-}$, given by the eigensubspaces of the endomorphism $\omega_{\mathbb{C}}:=i^{k} e_{1} \cdots e_{m}$ to the eigenvalues \pm 1 . In the sequel, we can call $\omega_{\mathbb{C}}$ the "chirality operator" or the "complex volume element".

In case $m$ is odd, that is $m=2 k+1$, the Clifford algebra $\mathbb{C} \ell(V)$ is isomorphic to $\mathcal{M}\left(2^{k} ; \mathbb{C}\right) \oplus$ $\mathcal{M}\left(2^{k} ; \mathbb{C}\right)$. And thus, we obtain two $2^{k}$-dimensional irreducible spinor modules $\mathbb{S}_{2 k+1}^{0}$ and $\mathbb{S}_{2 k+1}^{1}$ if we project the Clifford multiplication onto the first and second component respectively. Similar to the splitting in even dimensions, the two modules $\mathbb{S}_{2 k+1}^{0}$ and $\mathbb{S}_{2 k+1}^{1}$ can be distinguished by the action of the chirality operator $\omega_{\mathbb{C}}:=i^{k+1} e_{1} \cdots e_{m}$ in the sense that on $\mathbb{S}_{2 k+1}^{j}$ it acts as $(-1)^{j}, j=0,1$. It will cause no confusion if we simply identify $\mathbb{S}_{2 k+1}^{0}$ and $\mathbb{S}_{2 k+1}^{1}$ as the same vector space, that is $\mathbb{S}_{2 k+1}=\mathbb{S}_{2 k+1}^{0}=\mathbb{S}_{2 k+1}^{1}$, and equip them with Clifford multiplications of opposite sign. 
Now let $V$ and $W$ be two oriented Euclidean spaces with $\operatorname{dim} V=m_{1}$ and $\operatorname{dim} W=m_{2}$. We denote $\mathbb{C} \ell(V)$ and $\mathbb{C} \ell(W)$ the associated Clifford algebras of $V$ and $W$ respectively. By abuse of notation, we use the same symbol "." for the Clifford multiplication in $\mathbb{C} \ell(V), \mathbb{C} \ell(W)$ and in their representations. As is well known, the Clifford algebra of the sum of two vector spaces is the $\mathbb{Z}_{2}$-graded tensor product of the Clifford algebras of the two summands, that is $\mathbb{C} \ell(V \oplus W)=\mathbb{C} \ell(V) \widehat{\otimes} \mathbb{C} \ell(W)$ (see [34]). Therefore, we can construct the spinor module of $V \oplus W$ from those of $V$ and $W$ as

$$
\mathbb{S}_{m_{1}+m_{2}}=\left\{\begin{array}{cl}
\left(\mathbb{S}_{m_{1}} \oplus \mathbb{S}_{m_{1}}\right) \otimes \mathbb{S}_{m_{2}} & \text { both } m_{1} \text { and } m_{2} \text { are odd } \\
\mathbb{S}_{m_{1}} \otimes \mathbb{S}_{m_{2}} & m_{1} \text { is even. }
\end{array}\right.
$$

Here, we have excluded the case $m_{1}$ is odd and $m_{2}$ is even. This is simply because the place of $V$ and $W$ can be interchanged, which suggests that this case is symmetric to the case $m_{1}$ is even and $m_{2}$ is odd. As for the representation of Clifford multiplications on $\mathbb{S}_{m_{1}+m_{2}}$, let $\xi \in V$, $\zeta \in W, \varphi \in \mathbb{S}_{m_{2}}$ and $\psi=\psi_{1} \oplus \psi_{2} \in \mathbb{S}_{m_{1}} \oplus \mathbb{S}_{m_{1}}$ for both $m_{1}$ and $m_{2}$ are odd and $\psi \in \mathbb{S}_{m_{1}}$ otherwise, we set

$$
(\xi \oplus \zeta) \cdot(\psi \otimes \varphi)=(\xi \cdot \psi) \otimes \varphi+\left(\omega_{\mathbb{C}}^{V} \cdot \psi\right) \otimes(\zeta \cdot \varphi),
$$

where for both $m_{1}$ and $m_{2}$ odd we set $\xi \cdot \psi=\left(\xi \cdot \psi_{1}\right) \oplus\left(-\xi \cdot \psi_{2}\right)$ and $\omega_{\mathbb{C}}^{V} \cdot \psi=i\left(\psi_{2} \oplus-\psi_{1}\right)$. With this notation, one easily checks

$$
(\xi \oplus \zeta) \cdot(\xi \oplus \zeta) \cdot(\psi \otimes \varphi)=-|\xi \oplus \zeta|^{2}(\psi \otimes \varphi) .
$$

Thus $\mathbb{S}_{m_{1}+m_{2}}$ is a nontrivial $\mathbb{C} \ell(V \oplus W)$-module of dimension $2^{\left[\frac{m_{1}+m_{2}}{2}\right]}$. Moreover, in case $m_{1}+m_{2}$ is even, the splitting of $\mathbb{S}_{m_{1}+m_{2}}$ into half-spinor modules is given by

$$
\begin{gathered}
\mathbb{S}_{m_{1}+m_{2}}^{+}=\left\{(\psi \oplus \psi) \otimes \varphi: \psi \in \mathbb{S}_{m_{1}}, \varphi \in \mathbb{S}_{m_{2}}\right\}, \\
\mathbb{S}_{m_{1}+m_{2}}^{-}=\left\{(\psi \oplus-\psi) \otimes \varphi: \psi \in \mathbb{S}_{m_{1}}, \varphi \in \mathbb{S}_{m_{2}}\right\}
\end{gathered}
$$

for both $m_{1}$ and $m_{2}$ odd and

$$
\begin{aligned}
\mathbb{S}_{m_{1}+m_{2}}^{+} & =\left(\mathbb{S}_{m_{1}}^{+} \otimes \mathbb{S}_{m_{2}}^{+}\right) \oplus\left(\mathbb{S}_{m_{1}}^{-} \otimes \mathbb{S}_{m_{2}}^{-}\right), \\
\mathbb{S}_{m_{1}+m_{2}}^{-} & =\left(\mathbb{S}_{m_{1}}^{+} \otimes \mathbb{S}_{m_{2}}^{-}\right) \oplus\left(\mathbb{S}_{m_{1}}^{-} \otimes \mathbb{S}_{m_{2}}^{+}\right)
\end{aligned}
$$

for both $m_{1}$ and $m_{2}$ even.

Remark 2.1. The construction of the Clifford multiplication over $\mathbb{S}_{m_{1}+m_{2}}$ is a subtle issue. Comparing with the explicit formula (2.2), there are different ways to define the Clifford multiplication. For instance, in case both $m_{1}$ and $m_{2}$ are odd, let $\xi \in V, \zeta \in W, \varphi \in \mathbb{S}_{m_{2}}$ and $\psi=\psi_{1} \oplus \psi_{2} \in \mathbb{S}_{m_{1}} \oplus \mathbb{S}_{m_{1}}$, we can use the same expression of (2.2) but replace the previous definition of $\xi \cdot \psi$ with a new one $\xi \cdot \psi=\left(-\xi \cdot \psi_{2}\right) \oplus\left(-\xi \cdot \psi_{1}\right)$ (for a close reference, we refer [14]). In this setting, the half-spinor modules of $\mathbb{S}_{m_{1}+m_{2}}$ are

$$
\begin{aligned}
& \mathbb{S}_{m_{1}+m_{2}}^{+}=\left(\mathbb{S}_{m_{1}} \oplus\{0\}\right) \otimes \mathbb{S}_{m_{2}}, \\
& \mathbb{S}_{m_{1}+m_{2}}^{-}=\left(\{0\} \oplus \mathbb{S}_{m_{1}}\right) \otimes \mathbb{S}_{m_{2}}
\end{aligned}
$$

It would be better to understand that such changes in product formula give us equivalent definitions of Clifford multiplications. Indeed, due to the uniqueness of $\mathbb{C} \ell(V \oplus W)$, any definition of the Clifford multiplication on $\mathbb{S}_{m_{1}+m_{2}}$ can be identified with (2.2) via a vector space isomorphism. 
Next, let us consider the manifold setting. Let $\left(M_{1}, g^{(1)}\right)$ and $\left(M_{2}, g^{(2)}\right)$ be two oriented Riemannian manifolds of dimensions $m_{1}$ and $m_{2}$, respectively. We henceforth suppose that both manifolds are equipped with a fixed spin structure (for details about spin structures, we refer to [24,34] or to the well written self-contained introduction [28]). This induces a unique spin structure on the Riemannian product $\left(N=M_{1} \times M_{2}, g=g^{(1)} \oplus g^{(2)}\right)$. Indeed, let $\pi_{M_{1}}$ and $\pi_{M_{2}}$ denote the projections on $M_{1}$ and $M_{2}$, the tangent bundle of $N$ can be decomposed as

$$
T N=\pi_{M_{1}}^{*} T M_{1} \oplus \pi_{M_{2}}^{*} T M_{2} .
$$

For simplicity, we omit the projections and write $T N=T M_{1} \oplus T M_{2}$. And such splitting is orthogonal with respect to $g$. Hence the frame bundle of $N$ can be reduced to a $S O\left(m_{1}\right) \times$ $S O\left(m_{2}\right)$-principal bundle, and this is isomorphic to the product of the frame bundles over $M_{1}$ and $M_{2}$.

\subsection{The Dirac operator}

Fix the spin structures $\sigma_{M_{1}}$ and $\sigma_{M_{2}}$, let us consider the Clifford bundles (with Clifford multiplications) $\left(\mathbb{C l}\left(M_{1}\right),{ }_{g^{(1)}}\right),\left(\mathbb{C l}\left(M_{2}\right), \cdot_{g^{(2)}}\right)$ and spinor bundles $\mathbb{S}\left(M_{1}\right), \mathbb{S}\left(M_{2}\right)$ over $M_{1}$ and $M_{2}$ respectively. From the previous considerations in the algebraic settings, we know for the spinor bundles that

$$
\mathbb{S}(N)=\left\{\begin{array}{cl}
\left(\mathbb{S}\left(M_{1}\right) \oplus \mathbb{S}\left(M_{1}\right)\right) \otimes \mathbb{S}\left(M_{2}\right) & \text { both } m_{1} \text { and } m_{2} \text { are odd } \\
\mathbb{S}\left(M_{1}\right) \otimes \mathbb{S}\left(M_{2}\right) & m_{1} \text { is even. }
\end{array}\right.
$$

For $X \in T M_{1}, Y \in T M_{2}, \varphi \in \Gamma\left(\mathbb{S}\left(M_{2}\right)\right)$ and $\psi=\psi_{1} \oplus \psi_{2} \in \Gamma\left(\mathbb{S}\left(M_{1}\right) \oplus \mathbb{S}\left(M_{1}\right)\right)$ for both $m_{1}$ and $m_{2}$ odd and $\psi \in \Gamma\left(\mathbb{S}\left(M_{1}\right)\right)$ for $m_{1}$ even, we have

$$
(X \oplus Y) \cdot{ }_{g}(\psi \otimes \varphi)=\left(X \cdot g_{g^{(1)}} \psi\right) \otimes \varphi+\left(\omega_{\mathbb{C}}^{M_{1}} \cdot g_{g^{(1)}} \psi\right) \otimes\left(Y \cdot{ }_{g^{(2)}} \varphi\right)
$$

where in case $m_{1}$ and $m_{2}$ odd we set $X \cdot g_{g^{(1)}} \psi=\left(X \cdot_{g^{(1)}} \psi_{1}\right) \oplus\left(-X \cdot \cdot_{g^{(1)}} \psi_{2}\right)$ and $\omega_{\mathbb{C}}^{M_{1}} \cdot g_{g^{(1)}} \psi=$ $i\left(\psi_{2} \oplus-\psi_{1}\right)$.

Let $\nabla^{\mathbb{S}\left(M_{1}\right)}$ and $\nabla^{\mathbb{S}\left(M_{2}\right)}$ be the Levi-Civita connections on $\mathbb{S}\left(M_{1}\right)$ and $\mathbb{S}\left(M_{2}\right)$. By

$$
\nabla^{\mathbb{S}\left(M_{1}\right) \otimes \mathbb{S}\left(M_{2}\right)}=\nabla^{\mathbb{S}\left(M_{1}\right)} \otimes \operatorname{Id}_{\mathbb{S}\left(M_{2}\right)}+\operatorname{Id}_{\mathbb{S}\left(M_{1}\right)} \otimes \nabla^{\mathbb{S}\left(M_{2}\right)}
$$

we mean the tensor product connection on $\mathbb{S}\left(M_{1}\right) \otimes \mathbb{S}\left(M_{2}\right)$. If we take $\left\{X_{1}, \ldots, X_{m_{1}}\right\}$ a locally positively oriented orthonormal frame of $\left(M_{1}, g^{(1)}\right)$, then the Dirac operator on $M_{1}$ is (locally) defined by $D_{g^{(1)}}^{M_{1}}=\sum_{j=1}^{m_{1}} X_{j} \cdot g^{(1)} \nabla_{X_{j}}^{\mathbb{S}\left(M_{1}\right)}$. Similarly, if we take $\left\{Y_{1}, \ldots, Y_{m_{2}}\right\}$ a locally positively oriented orthonormal frame of $\left(M_{2}, g^{(2)}\right)$, we have $D_{g^{(2)}}^{M_{2}}=\sum_{j=1}^{m_{2}} Y_{j} \cdot g_{g^{(2)}} \nabla_{Y_{j}}^{\mathbb{S}\left(M_{2}\right)}$. Evidently, in the product setting, $\left\{X_{1} \oplus 0, \ldots, X_{m_{1}} \oplus 0,0 \oplus Y_{1}, \ldots, 0 \oplus Y_{m_{2}}\right\}$ is a local section of the frame bundle of $N$. Hence formula (2.3) yields

$$
\begin{aligned}
D_{g}^{N} & :=\sum_{j=1}^{m_{1}}\left(X_{j} \oplus 0\right) \cdot_{g^{(1)}} \nabla_{X_{j} \oplus 0}^{\mathbb{S}\left(M_{1}\right) \otimes \mathbb{S}\left(M_{2}\right)}+\sum_{j=1}^{m_{2}}\left(0 \oplus Y_{j}\right) \cdot_{g^{(2)}} \nabla_{0 \oplus Y_{j}}^{\mathbb{S}\left(M_{1}\right) \otimes \mathbb{S}\left(M_{2}\right)} \\
& =\tilde{D}_{g^{(1)}}^{M_{1}} \otimes \operatorname{Id}_{\mathbb{S}\left(M_{2}\right)}+\left(\omega_{\mathbb{C}}^{M_{1}} \cdot g^{(1)} \operatorname{Id}_{\mathbb{S}\left(M_{1}\right)}\right) \otimes D_{g^{(2)}}^{M_{2}}
\end{aligned}
$$


which defines the Dirac operator on $N=M_{1} \times M_{2}$, where $\tilde{D}_{g^{(1)}}^{M_{1}}=D_{g^{(1)}}^{M_{1}} \oplus-D_{g^{(1)}}^{M_{1}}$ if both $m_{1}$ and $m_{2}$ are odd and $\tilde{D}_{g^{(1)}}^{M_{1}}=D_{g^{(1)}}^{M_{1}}$ if $m_{1}$ is even.

For the case $m_{1}+m_{2}$ even, we have the decomposition $\mathbb{S}(N)=\mathbb{S}(N)^{+} \oplus \mathbb{S}(N)^{-}$and, moreover, when restrict $D_{g}^{N}$ on those half-spinor spaces we get $D_{g}^{N}: \Gamma\left(\mathbb{S}(N)^{ \pm}\right) \rightarrow \Gamma\left(\mathbb{S}(N)^{\mp}\right)$.

\subsection{Analysis on a product conformal structure}

In this section, we will consider our problem in details and we start with the case $N=M_{1} \times M_{2}$, $m_{1}=\operatorname{dim} M_{1} \geq 2$ and $m_{2}=\operatorname{dim} M_{2} \geq 1$. The case $m_{1}=m_{2}=1$, which corresponds to $N=S^{1} \times S^{1}$, will be discussed in Section 7 . From now on, in order to give unified expressions in odd and even cases, we will write it simply $\mathbb{S}(N)=\tilde{\mathbb{S}}\left(M_{1}\right) \otimes \mathbb{S}\left(M_{2}\right)$ with

$$
\tilde{\mathbb{S}}\left(M_{1}\right)=\left\{\begin{array}{cl}
\mathbb{S}\left(M_{1}\right) \oplus \mathbb{S}\left(M_{1}\right) & m_{1} \text { is odd } \\
\mathbb{S}\left(M_{1}\right) & m_{1} \text { is even }
\end{array}\right.
$$

and denote $\psi \otimes \varphi$ for a spinor field in $\mathbb{S}(N)$ when no confusion can arise.

To have a general view upon the problem, let us fix a function $\theta: M_{1} \rightarrow(0,+\infty)$ and consider the product conformal metric $g_{\ell}:=\ell^{2} \theta^{2} g^{(1)} \oplus g^{(2)}$, where $\ell>0$ is a parameter. According to the discussions in the previous section, we know for the Dirac operators that

$$
D_{g_{\ell}}^{N}=\tilde{D}_{\ell^{2} \theta^{2} g^{(1)}}^{M_{1}} \otimes \operatorname{Id}_{\mathbb{S}\left(M_{2}\right)}+\left(\overline{\omega_{\mathbb{C}}^{M_{1}}} \cdot_{\ell^{2} \theta^{2} g^{(1)}} \operatorname{Id}_{\tilde{\mathbb{S}}\left(M_{1}\right)}\right) \otimes D_{g^{(2)}}^{M_{2}}
$$

where $\overline{\omega_{\mathbb{C}}^{M_{1}}}$ denotes the chirality operator and " ${ }_{\ell^{2} \theta^{2} g^{(1)}}$ " denotes the Clifford multiplication on $M_{1}$ with respect to the conformal metric $\ell^{2} \theta^{2} g^{(1)}$ respectively.

Turning to the nonlinear problems, let us denote $|\cdot|_{\ell^{2} \theta^{2} g^{(1)}}$ and $|\cdot|_{g^{(2)}}$ the natural hermitian metrics on $\mathbb{S}\left(M_{1}\right)$ and $\mathbb{S}\left(M_{2}\right)$ respectively and $|\cdot|_{g_{\ell}}$ the induced metric on $\mathbb{S}(N)$. Set $n=$ $m_{1}+m_{2}$ and $n^{*}=\frac{2 n}{n-1}$, we can expand the spinorial Yamabe equation

$$
D_{g_{\ell}}^{N} \phi=|\phi|_{g_{\ell}}^{n^{*}-2} \phi, \quad \phi=\bar{\psi} \otimes \varphi \in \mathbb{S}(N)
$$

into

$$
\left(\tilde{D}_{\ell^{2} \theta^{2} g^{(1)}}^{M_{1}} \bar{\psi}\right) \otimes \varphi+\left(\overline{\omega_{\mathbb{C}}^{M_{1}}} \cdot_{\ell^{2} \theta^{2} g^{(1)}} \bar{\psi}\right) \otimes\left(D_{g^{(2)}}^{M_{2}} \varphi\right)=\left(|\bar{\psi}|_{\ell^{2} \theta^{2} g^{(1)}}|\varphi|_{g^{(2)}}\right)^{n^{*}-2} \bar{\psi} \otimes \varphi .
$$

We will now show how to dispense with the assumption on $\left(M_{2}, g^{(2)}, \sigma_{M_{2}}\right)$. In fact, if $M_{2}$ possesses a nontrivial eigenspinor $\varphi_{M_{2}}$ of constant length for some $\lambda \neq 0$, then by substituting $\bar{\psi} \otimes \varphi_{M_{2}}$ into (2.4) we get an equivalent problem

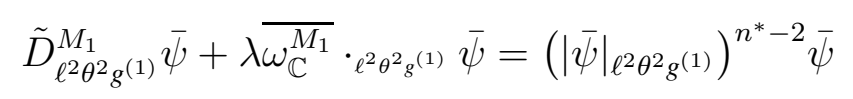

which is sitting on $M_{1}$. Here, we adopt the convention that $\lambda>0$ since (up to a change of orientation on $M_{1}$ ) the proof for $\lambda<0$ is exactly the same.

The following transformation formula describes how Dirac operators for conformally equivalent metrics are related (see [27,29]). 
Proposition 2.2. Let $g_{0}$ and $g=f^{2} g_{0}$ be two conformal metrics on a Riemannian spin $m$ manifold $M$. Then, there exists an isomorphism of vector bundles $F: \mathbb{S}\left(M, g_{0}\right) \rightarrow \mathbb{S}(M, g)$ which is a fiberwise isometry such that

$$
D_{g}^{M}(F(\psi))=F\left(f^{-\frac{m+1}{2}} D_{g_{0}}^{M}\left(f^{\frac{m-1}{2}} \psi\right)\right) .
$$

As a direct consequence, the equation (2.5) can be conformally transformed into

$$
\tilde{D}_{g^{(1)}}^{M_{1}} \psi+\lambda \ell \theta \omega_{\mathbb{C}}^{M_{1}} \dot{g}_{g^{(1)}} \psi=(\ell \theta)^{m_{1}-\frac{m_{1}-1}{2} n^{*}}|\psi|_{g^{(1)}}^{n^{*}-2} \psi
$$

Moreover, we can consider the rescaling $\psi \mapsto \ell^{-\frac{m_{1}-1}{2}} \psi$ in $\widetilde{\mathbb{S}}\left(M_{1}\right)$ and denote $\varepsilon=\ell^{-1}$ so that the above equation is equivalent to

$$
\varepsilon \tilde{D}_{g^{(1)}}^{M_{1}} \psi+\lambda \theta \omega_{\mathbb{C}}^{M_{1}} \cdot_{g^{(1)}} \psi=\theta^{m_{1}-\frac{m_{1}-1}{2} n^{*}}|\psi|_{g^{(1)}}^{n^{*}-2} \psi \quad \text { on } M_{1} .
$$

\subsection{Functional framework}

Our goal is to find solutions of (2.6) for varying $\varepsilon>0$. Notice that Eq. (2.6) is well-defined on $M_{1}$, and $n^{*}=\frac{2 n}{n-1}<\frac{2 m_{1}}{m_{1}-1}=m_{1}^{*}$. It is not necessary to carry the super- and sub-scripts in $\left(M_{1}, g^{(1)}\right), \tilde{D}_{g^{(1)}}^{M_{1}}$ and $\omega_{\mathbb{C}}^{M_{1}}$ during the proofs, hence in order to simplify the notation, we drop these super- and sub-scripts and to consider the model problem

$$
\varepsilon \tilde{D}_{g} \psi+a \omega_{\mathbb{C}} \cdot \psi=b|\psi|_{g}^{p-2} \psi
$$

on a spin $m$-manifold $(M, g, \sigma)$, where $a, b: M \rightarrow(0,+\infty)$ are functions at least $C^{1}$ smooth and $2<p<m^{*}:=\frac{2 m}{m-1}$. Unless otherwise stated, we will also drop the subscript of $|\cdot|_{g}$ on $\widetilde{\mathbb{S}}(M)$ for notation convenience.

For $q>1$, let us denote $L^{q}:=L^{q}(M, \tilde{\mathbb{S}}(M))$ which is defined as the completion of the space $\Gamma_{c}(\tilde{\mathbb{S}}(M)):=\{\psi \in \Gamma(\tilde{\mathbb{S}}(M)): \operatorname{supp}(\psi)$ is compact $\}$ with respect to the norm $|\cdot|_{q}^{q}:=$ $\int_{M}|\cdot|{ }^{q} d \operatorname{vol}_{g}$. Particularly, for $q=2$, we have $L^{2}$ is a Hilbert space with inner product $(\cdot, \cdot)_{2}=$ $\operatorname{Re} \int_{M}(\cdot, \cdot) d \operatorname{vol}_{g}$.

Let us set $A:=\varepsilon \tilde{D}_{g}+a \omega_{\mathbb{C}}$, our first point is to study the spectrum $\operatorname{Spec}(A)$ of $A$ in $L^{2}$. In fact, there is no difficulty to see that $A$ is self-adjoint and hence $\operatorname{Spec}(A) \subset \mathbb{R}$.

Lemma 2.3. For closed Riemannian spin manifold $(M, g)$,

(1) Spec $(A)$ is a closed subset of $\mathbb{R} \backslash\{0\}$ consisting of an unbounded discrete sequence of eigenvalues;

(2) each eigenspace of $A$ is finite-dimensional and consists of smooth sections;

(3) the eigenspaces of A form a complete orthonormal decomposition of $L^{2}$, that is,

$$
L^{2}=\bigoplus_{\lambda \in \operatorname{Spec}(A)} \operatorname{ker}(A-\lambda)
$$

(4) the set $\operatorname{Spec}(A)$ is symmetric about the origin. 
Proof. Let us set $a_{\text {min }}=\min _{M} a>0$ and write $a=\hat{a}+a_{\text {min }}$. Then, we can introduce the operator $\hat{A}:=\varepsilon \tilde{D}_{g}+\hat{a} \omega_{\mathbb{C}}$ and deduce

$$
\left(A^{2} \psi, \psi\right)_{2}=(\hat{A} \psi, \hat{A} \psi)_{2}+a_{m i n}^{2}|\psi|_{2}^{2}+2 a_{\min }(\hat{a} \psi, \psi)_{2} \geq a_{m i n}^{2}|\psi|_{2}^{2}
$$

This suggests $\operatorname{Spec}(A) \subset\left(-\infty,-a_{\text {min }}\right] \cup\left[a_{\text {min }},+\infty\right)$.

Notice that $\omega_{\mathbb{C}} \cdot$ and $\tilde{D}_{g}$ anticommute, thus when denote by $A_{\min }:=\varepsilon \tilde{D}_{g}+a_{\min } \omega_{\mathbb{C}}$ we get

$$
A_{\text {min }}^{2}=\varepsilon^{2} \tilde{D}_{g}^{2}+a_{\min }^{2}
$$

And this implies together with the spectral theorem of Dirac operators that the $L^{2}$-spectrum of $A_{\text {min }}$ is given by an unbounded discrete sequence of eigenvalues of finite multiplicity. Since the linear map $\psi \mapsto \hat{a} \omega_{\mathbb{C}} \cdot \psi$ is relatively compact with respect to $A_{\text {min }}$, we deduce that $A$ has compact resolvent. This proves (1). And immediately, the statements (2) and (3) follow from the classical spectral theory of elliptic self-adjoint operators.

As for the symmetry of $\operatorname{Spec}(A)$ about 0 , it straightforwardly follows from the splitting of the spinor bundle in the following sense. In case $m$ is odd, let $\psi=\psi_{1} \oplus \psi_{2}$ be an eigenspinor to an eigenvalue $\lambda \in \operatorname{Spec}(A)$, we have $\psi=\psi^{s}+\psi^{d}$ with

$$
\psi^{s}=\frac{\psi_{1}+\psi_{2}}{2} \oplus \frac{\psi_{1}+\psi_{2}}{2} \quad \text { and } \quad \psi^{d}=\frac{\psi_{1}-\psi_{2}}{2} \oplus-\frac{\psi_{1}-\psi_{2}}{2} .
$$

Since $A=\varepsilon \tilde{D}_{g}+a \omega_{\mathbb{C}}$ exchanges the above splitting of $\mathbb{S}(M) \oplus \mathbb{S}(M)$, we have

$$
\varepsilon \tilde{D}_{g} \psi^{s}+a \omega_{\mathbb{C}} \cdot \psi^{s}=\lambda \psi^{d} \quad \text { and } \quad \varepsilon \tilde{D}_{g} \psi^{d}+a \omega_{\mathbb{C}} \cdot \psi^{d}=\lambda \psi^{s}
$$

and therefore

$$
A\left(\psi^{s}-\psi^{d}\right)=-\lambda\left(\psi^{s}-\psi^{d}\right) .
$$

In case $m$ even, $\mathbb{S}(M)$ itself splits into $\mathbb{S}^{+}(M) \oplus \mathbb{S}^{-}(M)$ of eigenspaces of $\omega_{\mathbb{C}}$ and there is a representation of the Dirac operator as

$$
\tilde{D}_{g}=D_{g}=\left(\begin{array}{cc}
0 & \left.D_{g}\right|_{\mathbb{S}^{-}(M)} \\
\left.D_{g}\right|_{\mathbb{S}^{+}(M)} & 0
\end{array}\right): \Gamma\left(\mathbb{S}^{+}(M)\right) \oplus \Gamma\left(\mathbb{S}^{-}(M)\right) \rightarrow \Gamma\left(\mathbb{S}^{-}(M)\right) \oplus \Gamma\left(\mathbb{S}^{+}(M)\right) .
$$

One can pass from $\mathbb{S}^{+}(M)$ to $\mathbb{S}^{-}(M)$ by taking the same underlying vector bundle $\widetilde{\mathbb{S}}_{M}=$ $\mathbb{S}^{+}(M)=\mathbb{S}^{-}(M)$ and set

$$
\tilde{\mathbb{S}}_{M}^{1}:=\left\{(\psi,-i \psi): \psi \in \tilde{\mathbb{S}}_{M}\right\} \quad \text { and } \quad \tilde{\mathbb{S}}_{M}^{2}:=\left\{(\psi, i \psi): \psi \in \widetilde{\mathbb{S}}_{M}\right\}
$$

Then there is an isomorphism between vector bundles

$$
\tilde{\mathbb{S}}_{M} \oplus \tilde{\mathbb{S}}_{M} \rightarrow \tilde{\mathbb{S}}_{M}^{1} \oplus \tilde{\mathbb{S}}_{M}^{2}, \quad(\psi, 0) \mapsto \frac{1}{\sqrt{2}}(\psi,-i \psi) \quad \text { and } \quad(0, \psi) \mapsto \frac{1}{\sqrt{2}}(\psi, i \psi)
$$

so that the operator $A$ has the representation

$$
\tilde{D}_{g} \mapsto\left(\begin{array}{cc}
\left.D_{g}\right|_{\tilde{\mathbb{S}}} & 0 \\
0 & -\left.D_{g}\right|_{\tilde{\mathbb{S}}}
\end{array}\right) \quad \text { and } \quad \omega_{\mathbb{C}} \mapsto i\left(\begin{array}{cc}
0 & \operatorname{Id}_{\tilde{\mathbb{S}}} \\
-\operatorname{Id}_{\tilde{\mathbb{S}}} & 0
\end{array}\right)
$$

which is exactly the same as the case $m$ odd. Therefore, one easily checks $\operatorname{Spec}(A)$ is symmetric about 0 . 
From now on, we make the assumption that $(M, g)$ is closed. Then we can choose a complete orthonormal basis $\psi_{ \pm 1}, \psi_{ \pm 2}, \ldots$ of $L^{2}$ consisting of the eigenspinors of $A$, i.e. $A \psi_{ \pm k}=\lambda_{ \pm k} \psi_{ \pm k}$ and the spectrum $\operatorname{Spec}(A)$ will be denoted as

$$
\cdots \leq \lambda_{-2} \leq \lambda_{-1}<0<\lambda_{1} \leq \lambda_{2} \leq \cdots,
$$

where each eigenvalue appears with its multiplicity. Particularly, we have $\lambda_{k}=-\lambda_{-k}$ and $\left|\lambda_{ \pm k}\right| \rightarrow+\infty$ as $k \rightarrow \infty$.

From a variational point of view, to study Eq. (2.7), we need to define the unbounded operator $|A|^{s}: L^{2} \rightarrow L^{2}, s \geq 0$, by

$$
|A|^{s} \psi=\sum_{k=-\infty}^{\infty}\left|\lambda_{k}\right|^{s} \alpha_{k} \psi_{k}
$$

where $\psi=\sum_{k=-\infty}^{\infty} \alpha_{k} \psi_{k} \in L^{2}$. In this way, we can introduce the domain of $|A|^{s}$ in $L^{2}$ as

$$
\mathscr{H}^{s}:=\left\{\psi=\sum_{k=1}^{\infty} \alpha_{k} \psi_{k} \in L^{2}: \sum_{k=-\infty}^{\infty}\left|\lambda_{k}\right|^{2 s}\left|\alpha_{k}\right|^{2}<\infty\right\} .
$$

It is worth pointing out that $\mathscr{H}^{\frac{1}{2}}$ coincides with the Sobolev space of order $\frac{1}{2}$, that is $W^{\frac{1}{2}, 2}(M, \tilde{\mathbb{S}}(M))$ (see for instance [2,4]). Moreover, we can equip $\mathcal{H}:=\mathscr{H}^{\frac{1}{2}}$ an inner product

$$
\langle\psi, \varphi\rangle_{\varepsilon}:=\frac{1}{\varepsilon^{m}} \operatorname{Re} \int_{M}\left(|A|^{1 / 2} \psi,|A|^{1 / 2} \varphi\right) d \operatorname{vol}_{g}
$$

and the induced norm $\|\cdot\|_{\varepsilon}$ such that $\left(\mathcal{H},\langle\cdot, \cdot\rangle_{\varepsilon}\right)$ becomes a Hilbert space. Remark that, in the above notations, we have emphasized the dependence on the parameter $\varepsilon$ because it is already hidden in the operator $A$ and its spectrum. The dual space of $\mathcal{H}$ will be denoted by $\mathcal{H}^{*}=$ $W^{-\frac{1}{2}, 2}(M, \tilde{\mathbb{S}}(M))$. Identifying $\mathcal{H}$ with $\mathcal{H}^{*}$ we have $\langle\cdot, \cdot\rangle_{\varepsilon}$ can be used to denote the norm on $\mathcal{H}^{*}$.

On the Banach space $L^{q}, q>1$, we equip it a new norm

$$
|\psi|_{q, \varepsilon}=\left(\frac{1}{\varepsilon^{m}} \int_{M}|\psi|^{q} d \operatorname{vol}_{g}\right)^{\frac{1}{q}}
$$

Then, recall $m^{*}=\frac{2 m}{m-1}$, we get

Lemma 2.4. If $\varepsilon>0$ is small, then for any $q \in\left[2, m^{*}\right]$ the embedding $I d_{\mathcal{H}}:\left(\mathcal{H},\|\cdot\|_{\varepsilon}\right) \hookrightarrow$ $\left(L^{q},|\cdot|_{q, \varepsilon}\right)$ is a continuous map independent of $\varepsilon$, that is, there exists $c_{q}>0$ does not depend on $\varepsilon$ such that

$$
|\psi|_{q, \varepsilon} \leq c_{q}\|\psi\|_{\varepsilon} \quad \text { for all } \psi \in \mathcal{H}
$$

In particular, the embedding is compact for $q \in\left[2, m^{*}\right)$.

Proof. Our strategy is to use interpolation inequalities and we sketch the proof as follows. To begin with, we observe that, for $\psi \in \Gamma(\tilde{\mathbb{S}}(M))$,

$$
A^{2} \psi=\varepsilon^{2}\left(\tilde{D}_{g}\right)^{2} \psi+a^{2} \psi+\varepsilon \nabla a \cdot \omega_{\mathbb{C}} \cdot \psi .
$$


When denoted by $S_{c a l}$ the scalar curvature of $(M, g)$, by Schrödinger-Lichnerowicz formula, we have

$$
\left(\tilde{D}_{g}\right)^{2}=\nabla^{*} \nabla+\frac{1}{4} S c a l_{g}
$$

where $\nabla^{*} \nabla$ is the standard connection Laplacian. Hence we get

$$
\begin{aligned}
\| A|\psi|_{2}^{2} & =|A \psi|_{2}^{2}=\int_{M}\left(A^{2} \psi, \psi\right) d \operatorname{vol}_{g} \\
& =\int_{M} \varepsilon^{2}|\nabla \psi|^{2}+\left(a^{2}+\frac{\varepsilon^{2}}{4} S c a l_{g}\right)|\psi|^{2}+\varepsilon\left(\nabla a \cdot \omega_{\mathbb{C}} \cdot \psi, \psi\right) d \operatorname{vol}_{g}
\end{aligned}
$$

for all $\psi \in \Gamma(\tilde{\mathbb{S}}(M))$.

Since $M$ is closed, the curvature function $S_{c a l}$ is bounded. Noting that $a>0$ is of class $C^{1}$, we assert from (2.9) that, for small values of $\varepsilon$, the space $\mathscr{H}^{1}$ coincides with the standard Sobolev space $W^{1,2}(M, \tilde{\mathbb{S}}(M))$ and, for each fixed $\varepsilon$, the map $\psi \mapsto \| A|\psi|_{2}$ defines an equivalent norm on $W^{1,2}(M, \widetilde{\mathbb{S}}(M))$.

Recalling the classical Sobolev embedding theorems, we can conclude that there exists a positive constant $C$ (which depends on the dimension) such that

$$
\left(\int_{M}|\psi|^{\frac{2 m}{m-2}} d \operatorname{vol}_{g}\right)^{\frac{m-2}{m}} \leq C \int_{M}\left(|\nabla \psi|^{2}+|\psi|^{2}\right) d \operatorname{vol}_{g}
$$

for all $\psi \in W^{1,2}(M, \tilde{\mathbb{S}}(M))$. One easily gets

$$
\left(\frac{1}{\varepsilon^{m}} \int_{M}|\psi|^{\frac{2 m}{m-2}} d \operatorname{vol}_{g}\right)^{\frac{m-2}{m}} \leq \frac{C}{\varepsilon^{m}} \int_{M}\left(\varepsilon^{2}|\nabla \psi|^{2}+|\psi|^{2}\right) d \operatorname{vol}_{g}
$$

for all $\psi \in \mathscr{H}^{1}$ provided that $\varepsilon$ is small.

In the next, we use the following notation for a different norm of $\psi \in W^{1,2}(M, \tilde{\mathbb{S}}(M))$

$$
\|\psi\|_{1,2 ; \varepsilon}^{2}:=\frac{1}{\varepsilon^{m}} \int_{M}\left(\varepsilon^{2}|\nabla \psi|^{2}+|\psi|^{2}\right) d \operatorname{vol}_{g} .
$$

Then, consider the interpolation couples

$$
\left(\mathscr{H}^{1},\|\cdot\|_{1,2 ; \varepsilon}\right) \hookrightarrow\left(L^{m^{*}},|\cdot|_{m^{*}, \varepsilon}\right)
$$

and

$$
\left(\mathscr{H}^{0},|\cdot|_{2, \varepsilon}\right) \hookrightarrow\left(L^{2},|\cdot|_{2, \varepsilon}\right),
$$

we can easily assert from the Calderón-Lions interpolation theorem [40] that the embedding constant for $\left(\mathscr{H}^{\frac{1}{2}},\|\cdot\|_{\varepsilon}\right) \hookrightarrow\left(L^{m^{*}},|\cdot|_{m^{*}, \varepsilon}\right)$ is independent of $\varepsilon$.

For the compactness, we only need to point out that the embedding $W^{1,2}(M, \tilde{\mathbb{S}}(M)) \hookrightarrow L^{q}$ is compact for $q \in\left[2, m^{*}\right)$. Therefore, by the interpolation theorem again, we have $\mathcal{H}=\mathscr{H}^{\frac{1}{2}} \hookrightarrow$ $L^{q}$ is compact for $q \in\left[2, m^{*}\right)$, which completes the proof. 
Remark 2.5. Lemma 2.4 gives rise to a question on the $\varepsilon$-dependence of the embedding constant $c_{q}$ for large values of $\varepsilon$. This is quite involved. Since the form domain of the operator $A$ is the fractional Sobolev space $W^{\frac{1}{2}, 2}(M, \tilde{\mathbb{S}}(M))$, the embedding $\mathcal{H} \hookrightarrow L^{q}, q \in\left[2, m^{*}\right]$ exists in any circumstance. However, from the definition of the norm $\|\cdot\|_{\varepsilon}$ in (2.8), one can not get the explicit dependence on the parameter $\varepsilon$. Moreover, by the Schrödinger-Lichnerowicz formula and formula (2.9), we see that, for large $\varepsilon$, the interaction between the function $a$ and the scalar curvature $S c a l_{g}$ enters into play. In this situation, the embedding constant for $W^{1,2}(M, \tilde{\mathbb{S}}(M)) \hookrightarrow L^{m^{*}}$ is $\varepsilon$-dependent when $S c a l_{g}$ possesses certain negative parts. This fact will probably impact the embedding constant of $\mathcal{H}$ into $L^{m^{*}}$.

Recall that we have an $(\cdot, \cdot)_{2}$-orthogonal decomposition

$$
L^{2}=L_{\varepsilon}^{+} \oplus L_{\varepsilon}^{-}, \quad \psi=\psi^{+}+\psi^{-}
$$

with

$$
L_{\varepsilon}^{+}:=\overline{\bigoplus_{k=1}^{+\infty} \operatorname{ker}\left(A-\lambda_{k}\right)} \text { and } L_{\varepsilon}^{-}:=\overline{\bigoplus_{k=-1}^{-\infty} \operatorname{ker}\left(A-\lambda_{k}\right)}
$$

so that $A$ is positive definite on $L_{\varepsilon}^{+}$and negative definite on $L_{\varepsilon}^{-}$. Then, this leads to the orthogonal decomposition of $\mathcal{H}$ with respect to the inner product $\langle\cdot, \cdot\rangle_{\varepsilon}$ as

$$
\mathcal{H}=\mathcal{H}_{\varepsilon}^{+} \oplus \mathcal{H}_{\varepsilon}^{-}, \quad \mathcal{H}_{\varepsilon}^{ \pm}=\mathcal{H} \cap L_{\varepsilon}^{ \pm}
$$

With the above notations, we have Eq. (2.7) is the Euler-Lagrange equation of the functional

$$
\begin{aligned}
\mathcal{L}_{\varepsilon}(\psi) & =\frac{1}{\varepsilon^{m}} \int_{M}\left(\frac{1}{2}(A \psi, \psi)-\frac{b}{p}|\psi|^{p}\right) d \operatorname{vol}_{g} \\
& =\frac{1}{2}\left(\left\|\psi^{+}\right\|_{\varepsilon}^{2}-\left\|\psi^{-}\right\|_{\varepsilon}^{2}\right)-\frac{1}{\varepsilon^{m} p} \int_{M} b|\psi|^{p} d \operatorname{vol}_{g}
\end{aligned}
$$

defined on $\mathcal{H}=\mathcal{H}_{\varepsilon}^{+} \oplus \mathcal{H}_{\varepsilon}^{-}$. And by Lemma 2.4, we have $\mathcal{L}_{\varepsilon} \in C^{2}(\mathcal{H}, \mathbb{R})$.

We emphasize that, by abuse of notation, we just simply write $\psi=\psi^{+}+\psi^{-}$for the orthogonal decomposition of $\mathcal{H}$ without mention its dependence on $\varepsilon$. However, one should always keep in mind that, for different values of $\varepsilon$, such decomposition of a spinor $\psi$ is different.

\section{Existence of solutions}

We shall now investigate the existence of a nontrivial solution for Eq. (2.7). This is equivalent to find nontrivial critical points of the functional $\mathcal{L}_{\varepsilon}$ in the Hilbert space $\mathcal{H}$. Fortunately, this is not a difficult task. Indeed, by noting that $p<m^{*}$, the compact embedding $\mathcal{H} \hookrightarrow L^{p}$ sheds light on several ways to obtain the existence issue.

A first approach is to construct, on a subspace of $\mathcal{H}$, a functional having a mountain pass geometry whose critical points are in one-to-one correspondence with critical points of $\mathcal{L}_{\varepsilon}$. This idea can be found in a paper of Buffoni, Jeanjean and Stuart in 1993 where the authors studied the solutions to the Choquard-Pekar equation in $\mathbb{R}^{3}$, see [22]. Compared with the problem (2.7), 
the strategy is to use a global Lyapunov-Schmidt reduction to control the part of the solutions in the space $\mathcal{H}_{\varepsilon}^{-}$. This will lead to study a functional defined only on $\mathcal{H}_{\varepsilon}^{+}$. Then a direct application of the Mountain Pass Theorem gives the existence of a critical point. Such reduction argument essentially requires the super-quadratic part to be strictly convex and $C^{2}$ smooth. This is automatically satisfied in our situation since the the super-quadratic part in $\mathcal{L}_{\varepsilon}$ more or less behaves like the $L^{p}$-integral.

This approach was extended by several papers to study different nonlinear PDE problems. Typical (but not comprehensive) results can be found in [1] where Schrödinger equations with periodic potentials and in [37, 38] where semiclassical Hamiltonian elliptic systems were studied.

A second approach devotes in searching directly critical points of indefinite functionals. Benci and Rabinowitz [16] opened this route. They constructed deformations having special representations by solving appropriate differential equations approximately by time discretization. This approach was subsequently improved by Hofer in [30]. Several applications are exhibited in the study of periodic solutions of the one-dimensional wave equation, in the study of periodic solutions of Hamiltonian systems of ordinary differential equations, in the existence theory of systems of elliptic equations, in the study of resonance problems of the Landesman and Lazer type, etc.

Substantial improvements along this approach were made by Kryszewski and Szulkin [32]. The authors built an abstract linking theorem to obtain critical points of a strongly indefinite functional. And applications of this linking theorem give rise to the existence of a solution to nonlinear Schrödinger equations with periodic potentials and some general nonlinearities. The convexity of the super-quadratic part is not required and the energy functional is of $C^{1}$. This approach was subsequently refined in the work of Bartsch and Ding [15] via a general setting on Banach spaces.

The third approach (using the fundamental idea in calculus of variations) is to consider a constrained variational structure. Specifically, to our model problem (2.7), the procedure is to construct, on the unit sphere of $\mathcal{H}_{\varepsilon}^{+}$, a map $\chi_{\varepsilon}$ in $\mathcal{H}$ such that the composition $\mathcal{L}_{\varepsilon} \circ \chi_{\varepsilon}$ is of $C^{1}$ smooth and all its critical points on the unit sphere correspond to solutions of (2.7). This formulation was developed by Szulkin and Weth [43], and this idea can be viewed as a refinement of the first approach in the sense that $\chi_{\varepsilon}$ can be understand as a normalized reduction map of $\mathcal{L}_{\varepsilon}$ to $\mathcal{H}_{\varepsilon}^{+}$. However, being differently, the advantage of this approach is to remove the convexity and to give the most simplified characterization of the critical value.

Here, owning to the original face of the problem (2.7), we adopt the first approach mentioned above to obtain the existence results. The basic reason, which tempts us into choosing this approach, is that we shall use the $C^{2}$ property and the reduction procedure to go further to get some useful estimate on the critical levels so that we could draw more information on our geometric objects.

Let's begin with the following compactness result of the functional $\mathcal{L}_{\varepsilon}$. Since the proof is classical, we simply omit it here.

Lemma 3.1. For each $\varepsilon>0$ small, $\mathcal{L}_{\varepsilon}$ satisfies the $(P . S .)_{c}$-condition for $c \geq 0$, that is,

$$
\left.\begin{array}{l}
\mathcal{L}_{\varepsilon}\left(\psi_{n}\right) \rightarrow c \\
\mathcal{L}_{\varepsilon}^{\prime}\left(\psi_{n}\right) \rightarrow 0
\end{array}\right\} \Rightarrow\left\{\psi_{n}\right\} \text { possesses a convergent subsequence in } \mathcal{H}
$$


Moreover, $\psi_{n} \rightarrow 0$ if and only if $c=0$.

Now, for the functionals $\mathcal{L}_{\varepsilon}$, we have

Proposition 3.2. For each $\varepsilon>0$ small,

(1) there exists $g_{\varepsilon} \in C^{1}\left(\mathcal{H}_{\varepsilon}^{+}, \mathcal{H}_{\varepsilon}^{-}\right)$such that

$$
\forall w \in \mathcal{H}_{\varepsilon}^{-}, w \neq g_{\varepsilon}(u) \Rightarrow \mathcal{L}_{\varepsilon}(u+w)<\mathcal{L}_{\varepsilon}\left(u+g_{\varepsilon}(u)\right),
$$

in particular,

$$
\left\|g_{\varepsilon}(u)\right\|_{\varepsilon}^{2} \leq \frac{2}{\varepsilon^{m} p} \int_{M} b|u|^{p} d \mathrm{vol}_{g}
$$

and $\mathcal{L}_{\varepsilon}^{\prime}\left(u+g_{\varepsilon}(u)\right)[w] \equiv 0$ for all $w \in \mathcal{H}_{\varepsilon}^{-}$;

(2) denoted by

$$
I_{\varepsilon}: \mathcal{H}_{\varepsilon}^{+} \rightarrow \mathbb{R}, \quad I_{\varepsilon}(u)=\mathcal{L}_{\varepsilon}\left(u+g_{\varepsilon}(u)\right),
$$

if $\left\{u_{n}\right\}$ is a (P.S.)-sequence for $I_{\varepsilon}$ then $\left\{u_{n}+g_{\varepsilon}\left(u_{n}\right)\right\}$ is a (P.S.)-sequence for $\mathcal{L}_{\varepsilon}$;

(3) there exists $\psi_{\varepsilon} \in \mathcal{H}$ such that $\mathcal{L}_{\varepsilon}^{\prime}\left(\psi_{\varepsilon}\right)=0$ and $\psi_{\varepsilon} \neq 0$.

Proof. By Lemma 3.1, we may apply the arguments in [22, Section 2] to get the existence of one critical point for $I_{\varepsilon}$ of Mountain Pass type.

Next we are intend to give a characterization of the critical point $\psi_{\varepsilon}$ obtained in the above proposition.

Lemma 3.3. For every $u \in \mathcal{H}_{\varepsilon}^{+} \backslash\{0\}$, the map $I_{\varepsilon, u}: \mathbb{R} \rightarrow \mathbb{R}, I_{\varepsilon, u}(t)=I_{\varepsilon}(t u)$, is of class $C^{2}$ and satisfies

$$
I_{\varepsilon, u}^{\prime}(t)=0, t>0 \quad \Longrightarrow \quad I_{\varepsilon, u}^{\prime \prime}(t)<0 .
$$

Moreover $I_{\varepsilon, u}(0)=I_{\varepsilon, u}^{\prime}(0)=0, I_{\varepsilon, u}^{\prime \prime}(0)>0$.

Proof. In order to see this, we compute $I_{\varepsilon, u}^{\prime}(t)=\mathcal{L}_{\varepsilon}^{\prime}\left(t u+g_{\varepsilon}(t u)\right)[u]$ which suggests that $I_{\varepsilon, u}$ is $C^{2}$. As we can see, the implication is equivalent to:

$$
I_{\varepsilon}^{\prime}(u)[u]=0, u \neq 0 \quad \Longrightarrow \quad I_{\varepsilon}^{\prime \prime}(u)[u, u]<0 .
$$

For simplicity, let us denote $\Psi_{\varepsilon}: \mathcal{H} \rightarrow \mathbb{R}$ by $\Psi_{\varepsilon}(\psi)=\frac{1}{\varepsilon^{m} p} \int_{M} b|\psi|^{p} d \operatorname{vol}_{g}$ and set $\psi=u+g_{\varepsilon}(u)$ and $\chi=g_{\varepsilon}^{\prime}(u)[u]-g_{\varepsilon}(u)$. By using $\left.\mathcal{L}_{\varepsilon}^{\prime}\left(u+g_{\varepsilon}(u)\right)\right|_{\mathcal{H}_{\varepsilon}^{-}} \equiv 0$, we have (3.1) is a consequence of the following computation:

$$
\begin{aligned}
I_{\varepsilon}^{\prime \prime}(u)[u, u]= & \mathcal{L}_{\varepsilon}^{\prime \prime}(\psi)\left[u+g_{\varepsilon}^{\prime}(u)[u], u\right]=\mathcal{L}_{\varepsilon}^{\prime \prime}(\psi)[\psi+\chi, \psi+\chi] \\
= & \mathcal{L}_{\varepsilon}^{\prime \prime}(\psi)[\psi, \psi]+2 \mathcal{L}_{\varepsilon}^{\prime \prime}(\psi)[\psi, \chi]+\mathcal{L}_{\varepsilon}^{\prime \prime}(\psi)[\chi, \chi] \\
= & I_{\varepsilon}^{\prime}(u)[u]+\left(\Psi_{\varepsilon}^{\prime}(\psi)[\psi]-\Psi_{\varepsilon}^{\prime \prime}(\psi)[\psi, \psi]\right)+2\left(\Psi_{\varepsilon}^{\prime}(\psi)[\chi]-\Psi_{\varepsilon}^{\prime \prime}(\psi)[\psi, \chi]\right) \\
& \quad-\Psi_{\varepsilon}^{\prime \prime}(\psi)[\chi, \chi]-\|\chi\|_{\varepsilon}^{2} \\
\leq & I_{\varepsilon}^{\prime}(u)[u]-\frac{1}{\varepsilon^{m}} \frac{p-2}{p-1} \int_{M} b|\psi|^{p} d \operatorname{vol}_{g}-\|\chi\|_{\varepsilon}^{2}
\end{aligned}
$$


A natural constraint for $I_{\varepsilon}$ is to consider the associated Nehari manifold:

$$
\mathscr{N}_{\varepsilon}:=\left\{u \in \mathcal{H}_{\varepsilon}^{+} \backslash\{0\}: I_{\varepsilon}^{\prime}(u)[u]=0\right\} .
$$

By Lemma 3.3 this is a smooth submanifold of codimension 1 in $\mathcal{H}_{\varepsilon}^{+}$. And consequently, the critical point found in Proposition 3.2 (3) can be characterized by

$$
\gamma_{\varepsilon}:=\mathcal{L}_{\varepsilon}\left(\psi_{\varepsilon}\right)=\inf _{u \in \mathcal{H}_{\varepsilon}^{+} \backslash\{0\}} \max _{\psi \in \mathbb{R} u \oplus \mathcal{H}_{\varepsilon}^{-}} \mathcal{L}_{\varepsilon}(\psi)=\inf _{u \in \mathcal{H}_{\varepsilon}^{+} \backslash\{0\}} \max _{t>0} I_{\varepsilon}(t u)=\inf _{u \in \mathcal{N}_{\varepsilon}} I_{\varepsilon}(u) .
$$

For later purpose, it is worth to point out that, by Lemma 2.4, there holds

$$
I_{\varepsilon}(t u) \geq \frac{t^{2}}{2}\|u\|_{\varepsilon}^{2}-\frac{c_{p}^{p} t^{p}}{p} \max b\|u\|_{\varepsilon}^{p} \quad \forall u \in \mathcal{H}_{\varepsilon}^{+} \backslash\{0\}, \forall t>0 .
$$

Hence there exists $\tau_{0}>0$ independent of $\varepsilon$ such that $\gamma_{\varepsilon} \geq \tau_{0}$.

In what follows, we intend to pass to the limit $\varepsilon \rightarrow 0$ and consider the convergence of the min-max level $\gamma_{\varepsilon}$. The idea is to use certain test spinors in the functional $\mathcal{L}_{\varepsilon}$. For this purpose, first of all, we need to establish an upper bound estimate. Without loss of generality, we assume that $\left\{\phi_{\varepsilon}\right\} \subset \mathcal{H}$ is an arbitrary sequence such that

$$
c_{1} \leq \mathcal{L}_{\varepsilon}\left(\phi_{\varepsilon}\right) \leq c_{2} \quad \text { and } \quad\left\|\mathcal{L}_{\varepsilon}^{\prime}\left(\phi_{\varepsilon}\right)\right\|_{\varepsilon} \rightarrow 0
$$

as $\varepsilon \rightarrow 0$ for some constants $c_{1}, c_{2}>0$. Here, we have identified the dual space $\mathcal{H}^{*}$ with $\mathcal{H}$.

Lemma 3.4. Under (3.5), we have

(1) $\left\|\phi_{\varepsilon}\right\|_{\varepsilon}$ is uniformly bounded in $\varepsilon$;

(2) $\left\|\phi_{\varepsilon}^{-}-g_{\varepsilon}\left(\phi_{\varepsilon}^{+}\right)\right\|_{\varepsilon} \leq O\left(\left\|\mathcal{L}_{\varepsilon}^{\prime}\left(\phi_{\varepsilon}\right)\right\|_{\varepsilon}\right)$ as $\varepsilon \rightarrow 0$;

(3) $I_{\varepsilon}^{\prime}\left(\phi_{\varepsilon}^{+}\right) \rightarrow 0$ as $\varepsilon \rightarrow 0$ in the dual space of $\mathcal{H}_{\varepsilon}^{+}$.

Proof. For the boundedness, we recall that Lemma 2.4 implies the embedding constant for $\mathcal{H} \hookrightarrow L^{p^{*}}$ is independent of $\varepsilon$, and hence the arguments in Lemma 3.1 can be employed.

For (2), let us first set $z_{\varepsilon}=\phi_{\varepsilon}^{+}+g_{\varepsilon}\left(\phi_{\varepsilon}^{+}\right)$and $v_{\varepsilon}=\phi_{\varepsilon}^{-}-g_{\varepsilon}\left(\phi_{\varepsilon}^{+}\right)$. Then we have $v_{\varepsilon} \in \mathcal{H}_{\varepsilon}^{-}$ and, by the definition of $g_{\varepsilon}$,

$$
0=\mathcal{L}_{\varepsilon}^{\prime}\left(z_{\varepsilon}\right)\left[v_{\varepsilon}\right]=-\left\langle g_{\varepsilon}\left(\phi_{\varepsilon}^{+}\right), v_{\varepsilon}\right\rangle_{\varepsilon}-\frac{1}{\varepsilon^{m}} \operatorname{Re} \int_{M} b\left|z_{\varepsilon}\right|^{p-2}\left(z_{\varepsilon}, v_{\varepsilon}\right) d \mathrm{vol}_{g} .
$$

Since $\left\|\mathcal{L}_{\varepsilon}^{\prime}\left(\phi_{\varepsilon}\right)\right\|_{\varepsilon} \rightarrow 0$ as $\varepsilon \rightarrow 0$, it follows that

$$
o\left(\left\|v_{\varepsilon}\right\|_{\varepsilon}\right)=\mathcal{L}_{\varepsilon}^{\prime}\left(\phi_{\varepsilon}\right)\left[v_{\varepsilon}\right]=-\left\langle\phi_{\varepsilon}^{-}, v_{\varepsilon}\right\rangle-\frac{1}{\varepsilon^{m}} \operatorname{Re} \int_{M} b\left|\phi_{\varepsilon}\right|^{p-2}\left(\phi_{\varepsilon}, v_{\varepsilon}\right) d \operatorname{vol}_{g} .
$$

And hence, we get

$$
\begin{gathered}
o\left(\left\|v_{\varepsilon}\right\|_{\varepsilon}\right)=\left\|v_{\varepsilon}\right\|_{\varepsilon}^{2}+\frac{1}{\varepsilon^{m}} \operatorname{Re} \int_{M} b\left|\phi_{\varepsilon}\right|^{p-2}\left(\phi_{\varepsilon}, v_{\varepsilon}\right) d \operatorname{vol}_{g} \\
-\frac{1}{\varepsilon^{m}} \operatorname{Re} \int_{M} b\left|z_{\varepsilon}\right|^{p-2}\left(z_{\varepsilon}, v_{\varepsilon}\right) d \operatorname{vol}_{g} .
\end{gathered}
$$


Remark that the map $\psi \rightarrow|\psi|^{p}$ is convex, we have

$$
\frac{1}{\varepsilon^{m}} \operatorname{Re} \int_{M} b\left|\phi_{\varepsilon}\right|^{p-2}\left(\phi_{\varepsilon}, v_{\varepsilon}\right) d \operatorname{vol}_{g}-\frac{1}{\varepsilon^{m}} \operatorname{Re} \int_{M} b\left|z_{\varepsilon}\right|^{p-2}\left(z_{\varepsilon}, v_{\varepsilon}\right) d \operatorname{vol}_{g} \geq 0 .
$$

Thus, from (3.6), we can infer that $\left\|v_{\varepsilon}\right\|_{\varepsilon} \leq O\left(\left\|\mathcal{L}_{\varepsilon}^{\prime}\left(\phi_{\varepsilon}\right)\right\|_{\varepsilon}\right)$ as $\varepsilon \rightarrow 0$.

In order to check $(3)$ we compute $I_{\varepsilon}^{\prime}\left(\phi_{\varepsilon}^{+}\right)=\mathcal{L}_{\varepsilon}^{\prime}\left(\phi_{\varepsilon}^{+}+g_{\varepsilon}\left(\phi_{\varepsilon}^{+}\right)\right)$, which implies $\left\|I_{\varepsilon}^{\prime}\left(\phi_{\varepsilon}^{+}\right)\right\|_{\varepsilon} \rightarrow 0$ as $\varepsilon \rightarrow 0$ is a direct consequence of the $C^{2}$ smoothness of $\mathcal{L}_{\varepsilon}$.

Next, let us introduce the functional $H_{\varepsilon}: \mathcal{H}_{\varepsilon}^{+} \rightarrow \mathbb{R}$ by $H_{\varepsilon}(u)=I_{\varepsilon}^{\prime}(u)[u]$. Then, it is clear that $H_{\varepsilon}$ is $C^{1}$ and its derivative is given by the formula

$$
H_{\varepsilon}^{\prime}(u)[w]=I_{\varepsilon}^{\prime}(u)[w]+I_{\varepsilon}^{\prime \prime}(u)[u, w]
$$

for $u, w \in \mathcal{H}_{\varepsilon}^{+}$. We also have $\mathscr{N}_{\varepsilon}=H_{\varepsilon}^{-1}(0) \backslash\{0\}$. Moreover, by (3.2), we have

$$
H_{\varepsilon}^{\prime}(u)[u] \leq 2 H_{\varepsilon}(u)-\frac{1}{\varepsilon^{m}} \frac{p-2}{p-1} \int_{M} b\left|u+g_{\varepsilon}(u)\right|^{p} d \operatorname{vol}_{g} .
$$

for any $u \in \mathcal{H}_{\varepsilon}^{+}$.

Proposition 3.5. For the sequence $\left\{\phi_{\varepsilon}\right\}$ in (3.5), there exists $\left\{t_{\varepsilon}\right\} \subset \mathbb{R}$ such that $t_{\varepsilon} \phi_{\varepsilon}^{+} \in \mathscr{N}_{\varepsilon}$ and $\left|t_{\varepsilon}-1\right| \leq O\left(\left\|I_{\varepsilon}^{\prime}\left(\phi_{\varepsilon}^{+}\right)\right\|_{\varepsilon}\right)$.

Proof. We begin with the observation: due to the condition (3.5) and Lemma 3.4 (3), there holds

$$
\liminf _{\varepsilon \rightarrow 0} \frac{1}{\varepsilon^{m}} \int_{M} b\left|\phi_{\varepsilon}^{+}+g_{\varepsilon}\left(\phi_{\varepsilon}^{+}\right)\right|^{p} d \operatorname{vol}_{g} \geq c_{0}
$$

for some constant $c_{0}>0$. Let us set $\eta_{\varepsilon}:(0, \infty) \rightarrow \mathbb{R}$ by $\eta_{\varepsilon}(t)=H_{\varepsilon}\left(t \phi_{\varepsilon}^{+}\right)$. One easily checks that $t \eta_{\varepsilon}^{\prime}(t)=H_{\varepsilon}^{\prime}\left(t \phi_{\varepsilon}^{+}\right)\left[t \phi_{\varepsilon}^{+}\right]$for all $t>0$. Hence, by (3.7) and Taylor's formula, we get

$$
t \eta_{\varepsilon}^{\prime}(t) \leq 2 \eta_{\varepsilon}(1)-\frac{1}{\varepsilon^{m}} \frac{p-2}{p-1} \int_{M} b\left|\phi_{\varepsilon}^{+}+g_{\varepsilon}\left(\phi_{\varepsilon}^{+}\right)\right|^{p} d \operatorname{vol}_{g}+C|t-1|
$$

for $t$ close to 1 with $C>0$ independent of $\varepsilon$. Here we have used the uniform boundedness of $\eta_{\varepsilon}^{\prime}(t)$ on bounded intervals.

Notice that $\eta_{\varepsilon}(1)=I_{\varepsilon}^{\prime}\left(\phi_{\varepsilon}^{+}\right)\left[\phi_{\varepsilon}^{+}\right] \rightarrow 0$ as $\varepsilon \rightarrow 0$, we conclude from (3.8) and (3.9) that there exists a small constant $\delta>0$ such that

$$
\eta_{\varepsilon}^{\prime}(t) \leq-\delta \text { for all } t \in(1-\delta, 1+\delta) \text { and } \varepsilon \text { small enough. }
$$

Moreover, from Lemma 3.3, we have $\eta_{\varepsilon}(1-\delta)>0$ and $\eta_{\varepsilon}(1+\delta)<0$. Then, by Inverse Function Theorem, $t_{\varepsilon}:=\eta_{\varepsilon}^{-1}(0)$ exists and

$$
u_{\varepsilon}:=t_{\varepsilon} \phi_{\varepsilon}^{+} \in \mathscr{N}_{\varepsilon} \cap \operatorname{span}\left\{\phi_{\varepsilon}^{+}\right\}
$$

is well-defined for all $\varepsilon$ small enough. Furthermore, since $\left|\eta_{\varepsilon}^{\prime}(t)^{-1}\right|$ is bounded by a constant, say $c_{\delta}>0$, on $(1-\delta, 1+\delta)$, we consequently get

$$
\left\|u_{\varepsilon}-\phi_{\varepsilon}^{+}\right\|_{\varepsilon}=\left|\eta_{\varepsilon}^{-1}(0)-\eta_{\varepsilon}^{-1}\left(H_{\varepsilon}\left(\phi_{\varepsilon}^{+}\right)\right)\right| \cdot\left\|\phi_{\varepsilon}^{+}\right\|_{\varepsilon} \leq c_{\delta}\left|H_{\varepsilon}\left(\phi_{\varepsilon}^{+}\right)\right| \cdot\left\|\phi_{\varepsilon}^{+}\right\|_{\varepsilon} .
$$

Now the conclusion follows from $H_{\varepsilon}\left(\phi_{\varepsilon}^{+}\right) \leq O\left(\left\|I_{\varepsilon}^{\prime}\left(\phi_{\varepsilon}^{+}\right)\right\|_{\varepsilon}\right)$. 
Corollary 3.6. For the sequence $\left\{\phi_{\varepsilon}\right\}$ in (3.5), there exists $\left\{u_{\varepsilon}\right\}$ such that $u_{\varepsilon} \in \mathscr{N}_{\varepsilon}$ and $\| \phi_{\varepsilon}-$ $u_{\varepsilon}-g_{\varepsilon}\left(u_{\varepsilon}\right) \|_{\varepsilon} \leq O\left(\left\|\mathcal{L}_{\varepsilon}^{\prime}\left(\phi_{\varepsilon}\right)\right\|_{\varepsilon}\right)$. Particularly,

$$
\max _{t>0} I_{\varepsilon}\left(t \phi_{\varepsilon}^{+}\right)=I_{\varepsilon}\left(u_{\varepsilon}\right) \leq \mathcal{L}_{\varepsilon}\left(\phi_{\varepsilon}\right)+O\left(\left\|\mathcal{L}_{\varepsilon}^{\prime}\left(\phi_{\varepsilon}\right)\right\|_{\varepsilon}^{2}\right) .
$$

Proof. To see this, let $u_{\varepsilon}=t_{\varepsilon} \phi_{\varepsilon}^{+}$be as in Proposition 3.5 and set $z_{\varepsilon}=\phi_{\varepsilon}^{+}+g_{\varepsilon}\left(\phi_{\varepsilon}^{+}\right)$. Then one obtains from Lemma 3.4 that

$$
\begin{aligned}
\left\|\phi_{\varepsilon}-u_{\varepsilon}-g_{\varepsilon}\left(u_{\varepsilon}\right)\right\|_{\varepsilon} & \leq\left\|\phi_{\varepsilon}-z_{\varepsilon}\right\|_{\varepsilon}+\left|t_{\varepsilon}-1\right| \cdot\left\|\phi_{\varepsilon}^{+}\right\|_{\varepsilon}+\left\|g_{\varepsilon}\left(\phi_{\varepsilon}^{+}\right)-g_{\varepsilon}\left(u_{\varepsilon}\right)\right\|_{\varepsilon} \\
& \leq O\left(\left\|\mathcal{L}_{\varepsilon}^{\prime}\left(\phi_{\varepsilon}\right)\right\|_{\varepsilon}\right)+O\left(\left\|I_{\varepsilon}^{\prime}\left(\phi_{\varepsilon}^{+}\right)\right\|_{\varepsilon}\right)
\end{aligned}
$$

where we have used an easily checked inequality

$$
\left\|g_{\varepsilon}\left(\phi_{\varepsilon}^{+}\right)-g_{\varepsilon}\left(u_{\varepsilon}\right)\right\|_{\varepsilon} \leq\left\|g_{\varepsilon}^{\prime}\left(\tau \phi_{\varepsilon}^{+}\right)\right\|_{\mathcal{H}_{\varepsilon}^{+} \rightarrow \mathcal{H}_{\varepsilon}^{-}} \cdot\left\|\phi_{\varepsilon}^{+}-u_{\varepsilon}\right\|_{\varepsilon}=O\left(\left|t_{\varepsilon}-1\right|\right)
$$

for some $\tau$ between $t_{\varepsilon}$ and 1 . Remark that $I_{\varepsilon}^{\prime}\left(\phi_{\varepsilon}^{+}\right)=\mathcal{L}_{\varepsilon}^{\prime}\left(z_{\varepsilon}\right)$, by using the $C^{2}$ smoothness of $\mathcal{L}_{\varepsilon}$, we have

$$
\left\|I_{\varepsilon}^{\prime}\left(\phi_{\varepsilon}^{+}\right)\right\|_{\varepsilon}=\left\|\mathcal{L}_{\varepsilon}^{\prime}\left(z_{\varepsilon}\right)\right\|_{\varepsilon} \leq\left\|\mathcal{L}_{\varepsilon}^{\prime}\left(\phi_{\varepsilon}\right)\right\|_{\varepsilon}+O\left(\left\|\phi_{\varepsilon}-z_{\varepsilon}\right\|_{\varepsilon}\right)=O\left(\left\|\mathcal{L}_{\varepsilon}^{\prime}\left(\phi_{\varepsilon}\right)\right\|_{\varepsilon}\right)
$$

This together with (3.10) implies

$$
\left\|\phi_{\varepsilon}-u_{\varepsilon}-g_{\varepsilon}\left(u_{\varepsilon}\right)\right\|_{\varepsilon} \leq O\left(\left\|\mathcal{L}_{\varepsilon}^{\prime}\left(\phi_{\varepsilon}\right)\right\|_{\varepsilon}\right) .
$$

Now, by Talyor's formula, we can obtain

$$
\begin{aligned}
\mathcal{L}_{\varepsilon}\left(\phi_{\varepsilon}\right) & =\mathcal{L}_{\varepsilon}\left(u_{\varepsilon}+g_{\varepsilon}\left(u_{\varepsilon}\right)\right)+\mathcal{L}_{\varepsilon}^{\prime}\left(u_{\varepsilon}+g_{\varepsilon}\left(u_{\varepsilon}\right)\right)\left[\phi_{\varepsilon}-u_{\varepsilon}-g_{\varepsilon}\left(u_{\varepsilon}\right)\right]+O\left(\left\|\mathcal{L}_{\varepsilon}^{\prime}\left(\phi_{\varepsilon}\right)\right\|_{\varepsilon}^{2}\right) \\
& =I_{\varepsilon}\left(u_{\varepsilon}\right)+I_{\varepsilon}^{\prime}\left(u_{\varepsilon}\right)\left[\phi_{\varepsilon}^{+}-u_{\varepsilon}\right]+O\left(\left\|\mathcal{L}_{\varepsilon}^{\prime}\left(\phi_{\varepsilon}\right)\right\|_{\varepsilon}^{2}\right) .
\end{aligned}
$$

Notice that $u_{\varepsilon}=t_{\varepsilon} \phi_{\varepsilon}^{+} \in \mathscr{N}_{\varepsilon}$, we have $I_{\varepsilon}^{\prime}\left(u_{\varepsilon}\right)\left[\phi_{\varepsilon}^{+}-u_{\varepsilon}\right] \equiv 0$ and this implies the last estimate.

As a immediate consequence of Corollary 3.6, we can show a explicit upper bound of $\gamma_{\varepsilon}$ if we find some test spinors $\left\{\phi_{\varepsilon}\right\}$ satisfying (3.5).

\section{Energy gap for solutions in Euclidean spaces: the bubbles}

We consider solutions to the equation

$$
\tilde{D}_{g_{\mathbb{R}} m} \psi+\nu \omega_{\mathbb{C}} \cdot \psi=\kappa|\psi|^{p-2} \psi \quad \text { on } \mathbb{R}^{m}
$$

belonging to the class $W^{\frac{1}{2}, 2}\left(\mathbb{R}^{m}, \tilde{\mathbb{S}}\left(\mathbb{R}^{m}\right)\right)$, where $\nu, \kappa>0$ are constants,

$$
\tilde{\mathbb{S}}\left(\mathbb{R}^{m}\right)=\left\{\begin{array}{cl}
\mathbb{S}\left(\mathbb{R}^{m}\right) \oplus \mathbb{S}\left(\mathbb{R}^{m}\right) & m \text { is odd } \\
\mathbb{S}\left(\mathbb{R}^{m}\right) & m \text { is even }
\end{array}\right.
$$

$\tilde{D}_{g_{\mathbb{R}^{m}}}=D_{g_{\mathbb{R}^{m}}} \oplus-D_{g_{\mathbb{R}} m}$ if $m$ is odd and $\tilde{D}_{g_{\mathbb{R}} m}=D_{g_{\mathbb{R}^{m}}}$ if $m$ is even. These solutions correspond to "bubbles" or test spinors for our variational problem. 
First of all, let us denote $A_{\nu}=\tilde{D}_{g_{\mathbb{R}} m}+\nu \omega_{\mathbb{C}}$. By a straightforward calculation we see that $A_{\nu}$ is a self-adjoint operator on $L^{2}$ and has its spectrum $\operatorname{Spec}\left(A_{\nu}\right)=(-\infty,-\nu] \cup[\nu,+\infty)$. Following Amann [3], denote $\left(E_{\lambda}\right)_{\lambda \in \mathbb{R}}$ the spectral resolution of $A_{\nu}$ and define the orthogonal projections by

$$
P_{\nu}=\int_{-\infty}^{0} d E_{\lambda}, \quad Q_{\nu}=\int_{0}^{\infty} d E_{\lambda} .
$$

Then the decomposition of $\mathcal{E}=W^{\frac{1}{2}, 2}\left(\mathbb{R}^{m}, \tilde{\mathbb{S}}\left(\mathbb{R}^{m}\right)\right)=\mathcal{E}_{\nu}^{+} \oplus \mathcal{E}_{\nu}^{-}$is induced by

$$
\mathcal{E}_{\nu}^{-}=\mathcal{E} \cap P_{\nu}\left(L^{2}\right) \quad \text { and } \quad \mathcal{E}_{\nu}^{+}=\mathcal{E} \cap Q_{\nu}\left(L^{2}\right) .
$$

We can introduce the following operators

$$
S_{\nu}=\int_{-\infty}^{0}|\lambda|^{\frac{1}{2}} d E_{\lambda} \quad \text { and } \quad T_{\nu}=\int_{0}^{\infty}|\lambda|^{\frac{1}{2}} d E_{\lambda} .
$$

We may now introduce a new inner product on $\mathcal{E}$ by the formula

$$
\langle\psi, \varphi\rangle_{\nu}=\operatorname{Re}\left(\left(S_{\nu}+T_{\nu}\right) \psi,\left(S_{\nu}+T_{\nu}\right) \varphi\right)_{2}, \quad \psi, \varphi \in \mathcal{E}
$$

and the corresponding norm $\|\cdot\|_{\nu}$. And we easily see that (4.1) is the Euler-Lagrange equation of the functional

$$
\Phi_{\nu \kappa}(\psi)=\frac{1}{2}\left(\left\|Q_{\nu} \psi\right\|_{\nu}^{2}-\left\|P_{\nu} \psi\right\|_{\nu}^{2}\right)-\frac{\kappa}{p}|\psi|_{p}^{p}
$$

Lemma 4.1. If $\left\{\psi_{n}\right\} \subset \mathcal{E}$ is a bounded sequence such that

$$
\Phi_{\nu \kappa}^{\prime}\left(\psi_{n}\right) \rightarrow 0 \text { and } \quad \liminf _{n \rightarrow \infty}\left|\psi_{n}\right|_{p}>0 .
$$

Then there exists $\psi \neq 0$ with $\Phi_{\nu \kappa}^{\prime}(\psi)=0$.

Proof. Let $B_{R}^{0}$ denote the open ball of radius $R$ centered at the origin. If

$$
\lim _{n \rightarrow \infty} \sup _{y \in \mathbb{R}^{m}} \int_{y+B_{R}^{0}}\left|\psi_{n}\right|^{2} d x=0, \quad \forall R>0,
$$

then by Lions' result [35] $\psi_{n} \rightarrow 0$ in $L^{q}$ for all $q \in\left(2, m^{*}\right)$ and therefore $\left|\psi_{n}\right|_{p} \rightarrow 0$, which is a contradiction.

Passing to a subsequence, we have

$$
\liminf _{n \rightarrow \infty} \int_{y_{n}+B_{R}^{0}}\left|\psi_{n}\right|^{2} d x>0
$$

for some $R>0$ and $\left\{y_{n}\right\} \subset \mathbb{R}^{m}$. Using the invariance of the operator $A_{\nu}$ under translations, we can find $R>0$ and a new sequence $\left\{\tilde{\psi}_{n}\right\}$ such that

$$
\Phi_{\nu \kappa}^{\prime}\left(\tilde{\psi}_{n}\right) \rightarrow 0 \text { and } \liminf _{n \rightarrow \infty} \int_{B_{R}^{0}}\left|\tilde{\psi}_{n}\right|^{2} d x>0 .
$$

Up to a subsequence if necessary, we have $\tilde{\psi}_{n} \rightarrow \psi$ and the compact embedding $\mathcal{E} \hookrightarrow L_{\text {loc }}^{2}$ shows that $\psi \neq 0$. Note that $\left|\tilde{\psi}_{n}\right|^{p-2} \tilde{\psi}_{n} \rightarrow|\psi|^{p-2} \psi$ in $L^{\frac{p}{p-1}}$, by taking the limit in $\Phi_{\nu \kappa}^{\prime}\left(\tilde{\psi}_{n}\right) \rightarrow 0$, we obtain $\Phi_{\nu \kappa}^{\prime}(\psi)=0$ as desired. 
Corollary 4.2. For each $\nu, \kappa>0$, there exists a nontrivial solution $\psi \in \mathcal{E}$ to Eq. (4.1).

Proof. By Lemma 4.1, this is a direct consequence of [22, Theorem 2.1].

Now we may define

$$
\gamma(\nu, \kappa)=\inf \left\{\Phi_{\nu \kappa}(\psi): \psi \in \mathcal{E} \backslash\{0\} \text { s.t. } \Phi_{\nu \kappa}^{\prime}(\psi)=0\right\} .
$$

Because the super-quadratic part in (4.2) is simply the $L^{p}$ norm, we easily see that $\gamma(\nu, \kappa)>0$ is attained. Particularly, by [22] and a similar argument as of Lemma 3.3, the following reduction principle holds.

Lemma 4.3. For each $\nu, \kappa>0$,

(1) there exists a $C^{1}$ map $h_{\nu \kappa}: \mathcal{E}_{\nu}^{+} \rightarrow \mathcal{E}_{\nu}^{-}$such that $\Phi_{\nu \kappa}\left(u+h_{\nu \kappa}(u)\right)=\max _{v \in \mathcal{E}_{\nu}^{-}} \Phi_{\nu \kappa}(u+v)$;

(2) denoted by $J_{\nu \kappa}(u)=\Phi_{\nu \kappa}\left(u+h_{\nu \kappa}(u)\right)$, then critical points of $J_{\nu \kappa}$ and $\Phi_{\nu \kappa}$ are in one-toone correspondence via the injective map $u \mapsto u+h_{\nu \kappa}(u)$;

(3) for each $u \in \mathcal{E}_{\nu}^{+} \backslash\{0\}$, the map $t \mapsto J_{\nu \kappa}(t u)$ has only one maximum on $(0,+\infty)$ and $\gamma(\nu, \kappa)=\inf _{u \in \mathcal{E}_{\nu}^{+} \backslash\{0\}} \max _{t>0} J_{\nu \kappa}(t u)$.

In the next step, we will study the behavior of the map $(\nu, \kappa) \mapsto \gamma(\nu, \kappa)$. Specifically, the monotonicity of $\gamma$ with respect to the two parameters is at the core of this paper.

Proposition 4.4. $\gamma(\nu, \kappa)=\nu^{-(m-1)+\frac{2}{p-2}} \kappa^{-\frac{2}{p-2}} \gamma(1,1)$.

Proof. In fact, taking $\rho>0$ as a parameter, we can assert that: $\psi$ is a nontrivial solution of Eq. (4.1) with energy $\gamma(\nu, \kappa)$ if and only if $\varphi(x)=\rho \psi(x / \nu)$ solves

$$
\tilde{D}_{g_{\mathbb{R}} m} \varphi+\omega_{\mathbb{C}} \cdot \varphi=\frac{\nu^{-1} \kappa}{\rho^{p-2}}|\varphi|^{p-2} \varphi \quad \text { on } \mathbb{R}^{m}
$$

with the energy

$$
\gamma\left(1, \frac{\nu^{-1} \kappa}{\rho^{p-2}}\right)=\nu^{m-1} \rho^{2} \gamma(\nu, \kappa) .
$$

Therefore, the conclusion follows easily by substituting $\rho=\left(\nu^{-1} \kappa\right)^{\frac{1}{p-2}}$.

Remark 4.5. Since $p \in\left(2, m^{*}\right)$, we have $-(m-1)+\frac{2}{p-2}>0$ and the value of $\gamma(\nu, \kappa)$ decreases as $\nu$ goes smaller and $\kappa$ goes larger.

\section{Bubbling analysis}

Let us recall that the coefficients $a, b$ in the model problem Eq. (2.7) are positive functions in the class $C^{1}(M)$. Motivated by Proposition 4.4, we introduce a potential function $\alpha: M \rightarrow \mathbb{R}$ as

$$
\alpha=a^{-(m-1)+\frac{2}{p-2}} b^{-\frac{2}{p-2}}
$$


and we write $\alpha_{\min }=\min _{M} \alpha$. The set of minimum points of $\alpha$ will be denoted by

$$
\mathcal{C}=\left\{\xi \in M: \alpha(\xi)=\alpha_{\min }\right\}
$$

Fix $\xi_{0} \in \mathcal{C}$ arbitrarily, we can denote $\nu_{0}=a\left(\xi_{0}\right)$ and $\kappa_{0}=b\left(\xi_{0}\right)$. Then, by Lemma 4.3, there exists $\psi_{0} \in \mathcal{E}$ such that

$$
\tilde{D}_{g_{\mathbb{R}} m} \psi_{0}+\nu_{0} \omega_{\mathbb{C}} \cdot \psi_{0}=\kappa_{0}\left|\psi_{0}\right|^{p-2} \psi_{0} \quad \text { on } \mathbb{R}^{m}
$$

and

$$
\Phi_{\nu_{0} \kappa_{0}}\left(\psi_{0}\right)=\gamma\left(\nu_{0}, \kappa_{0}\right) \equiv \alpha_{\min } \gamma(1,1) .
$$

Let $\eta \in C^{\infty}\left(\mathbb{R}^{m}\right)$ be such that $\eta(x)=1$ for $|x| \leq 1 / 2$ and $\eta(x)=0$ for $|x| \geq 1$. We define a spinor $\psi_{\varepsilon} \in \Gamma\left(\tilde{\mathbb{S}}\left(\mathbb{R}^{m}\right)\right)$ by

$$
\varphi_{\varepsilon}(x)=\eta_{\varepsilon}(x) \psi_{0}(x) \quad \text { where } \quad \eta_{\varepsilon}(x)=\eta\left(\varepsilon^{\frac{1}{2}} x\right) .
$$

Suppose $\xi_{0} \in V \subset M$ and let $\left(x_{1}, \ldots, x_{m}\right)$ be the normal coordinates given by the exponential map $\exp _{\xi_{0}}: U \subset T_{\xi_{0}} M \cong \mathbb{R}^{m} \rightarrow V, x \mapsto y=\exp _{\xi_{0}} x$. We define

$$
\mu_{\varepsilon}(x)=\exp _{\xi_{0}}(\varepsilon x)
$$

such that $\varepsilon|x|<i n j_{M}$, where $i n j_{M}>0$ is the injectivity radius of $M$.

Denoted by $B_{R}^{0}=\left\{x \in \mathbb{R}^{m}:|x|<R\right\}$, where $|\cdot|$ is the Euclidean norm in $\mathbb{R}^{m}$, we have a conformal equivalence $\left(B_{\varepsilon^{-1 / 2}}^{0}, \varepsilon^{-2} \mu_{\varepsilon}^{*} g\right) \cong\left(B_{\varepsilon^{1 / 2}}\left(\xi_{0}\right), g\right) \subset M$ for all $\varepsilon$ small.

For ease of notation, we set $g_{\varepsilon}=\varepsilon^{-2} \mu_{\varepsilon}^{*} g$. Writing the metric $g$ in geodesic normal coordinates centered at $\xi_{0}$, one immediately sees that $g_{\varepsilon}$ converges to the Euclidean metric in $C^{\infty}$-topology on $B_{\varepsilon^{-1 / 2}}^{0}$.

We point out here that, by using the idea of Bourguignon-Gauduchon trivialization [21] (see also [8]), the coordinate map $\mu_{\varepsilon}$ induces a bundle identification $\overline{\left(\mu_{\varepsilon}\right)_{*}}: \mathbb{S}_{x}\left(B_{\varepsilon^{-1 / 2}}^{0}, g_{\varepsilon}\right) \rightarrow$ $\mathbb{S}_{\mu_{\varepsilon}(x)}\left(B_{\varepsilon^{1 / 2}}\left(\xi_{0}\right), g\right)$. Hence we can define spinors on $B_{\varepsilon^{1 / 2}}\left(\xi_{0}\right)$ by

$$
\phi_{\varepsilon}:={\overline{\left(\mu_{\varepsilon}\right)_{*}}}_{\varphi_{\varepsilon}} \circ \mu_{\varepsilon}^{-1} \text {. }
$$

Then, by the transformation property of the Dirac operator under conformal change of the metric (see [27,29]), a straightforward calculation shows that

$$
\varepsilon \tilde{D}_{g} \phi_{\varepsilon}=\overline{\left(\mu_{\varepsilon}\right)_{*}} \circ\left(D_{g_{\varepsilon}} \varphi_{\varepsilon}\right) \circ \mu_{\varepsilon}^{-1},
$$

and moreover,

$$
\begin{aligned}
\frac{1}{\varepsilon^{m}} \int_{B_{\varepsilon^{1 / 2}}\left(\xi_{0}\right)} \varepsilon\left(\tilde{D}_{g} \phi_{\varepsilon}, \phi_{\varepsilon}\right) d \operatorname{vol}_{g} & =\int_{B_{\varepsilon^{-1 / 2}}^{0}}\left(D_{g_{\varepsilon}} \varphi_{\varepsilon}, \varphi_{\varepsilon}\right) d \operatorname{vol}_{g_{\varepsilon}}, \\
\frac{1}{\varepsilon^{m}} \int_{B_{\varepsilon^{1 / 2}}\left(\xi_{0}\right)}\left(\omega_{\mathbb{C} \cdot g} \phi_{\varepsilon}, \phi_{\varepsilon}\right) d \operatorname{vol}_{g} & =\int_{B_{\varepsilon^{-1 / 2}}^{0}}\left(\omega_{\mathbb{C}} \cdot g_{\varepsilon} \varphi_{\varepsilon}, \varphi_{\varepsilon}\right) d \operatorname{vol}_{g_{\varepsilon}}, \\
\frac{1}{\varepsilon^{m}} \int_{B_{\varepsilon^{1 / 2}}\left(\xi_{0}\right)}\left|\phi_{\varepsilon}\right|^{p} d \operatorname{vol}_{g} & =\int_{B_{\varepsilon^{-1 / 2}}^{0}}\left|\varphi_{\varepsilon}\right|^{p} d \operatorname{vol}_{g_{\varepsilon}},
\end{aligned}
$$

where $\omega_{\mathbb{C}} \cdot g$ and $\omega_{\mathbb{C}} \cdot g_{\varepsilon}$ denote the Clifford multiplication by the chirality operators with respect to the metric $g$ and $g_{\varepsilon}$ respectively. 
Lemma 5.1. $\left\|\mathcal{L}_{\varepsilon}^{\prime}\left(\phi_{\varepsilon}\right)\right\|_{\varepsilon} \rightarrow 0$ as $\varepsilon \rightarrow 0$.

Proof. Let $\varphi \in \mathcal{H}$ be an arbitrary test spinor, it follows that

$$
\mathcal{L}_{\varepsilon}\left(\phi_{\varepsilon}\right)[\varphi]=\frac{1}{\varepsilon^{m}} \operatorname{Re} \int_{M}\left(A \phi_{\varepsilon}, \varphi\right)-b\left|\phi_{\varepsilon}\right|^{p-2}\left(\phi_{\varepsilon}, \varphi\right) d \operatorname{vol}_{g} .
$$

Notice that $\phi_{\varepsilon}={\overline{\left(\mu_{\varepsilon}\right)_{*}}} \circ \varphi_{\varepsilon} \circ \mu_{\varepsilon}^{-1}$, we have

$$
\varepsilon \tilde{D}_{g} \phi_{\varepsilon}={\overline{\left(\mu_{\varepsilon}\right)_{*}}} \circ\left(\nabla \eta_{\varepsilon} \cdot_{\varepsilon} \psi_{0}\right) \circ \mu_{\varepsilon}^{-1}+{\overline{\left(\mu_{\varepsilon}\right)_{*}}} \circ\left(\eta_{\varepsilon} \tilde{D}_{g_{\varepsilon}} \psi_{0}\right) \circ \mu_{\varepsilon}^{-1}
$$

where $g_{\varepsilon}$ is the Clifford multiplication with respect to the metric $g_{\varepsilon}$. Substituting this into (5.9), we get

$$
\mathcal{L}_{\varepsilon}^{\prime}\left(\phi_{\varepsilon}\right)[\varphi]=l_{1}+l_{2}+l_{3}+l_{4}
$$

where

$$
\begin{aligned}
& l_{1}=\frac{1}{\varepsilon^{m}} \operatorname{Re} \int_{M}\left(\overline{\left(\mu_{\varepsilon}\right)_{*}} \circ\left(\nabla \eta_{\varepsilon} \cdot g_{\varepsilon} \psi_{0}\right) \circ \mu_{\varepsilon}^{-1}, \varphi\right) d \operatorname{vol}_{g} \\
& =\operatorname{Re} \int_{B_{\varepsilon^{-1 / 2}}^{0}}\left(\nabla \eta_{\varepsilon} \cdot g_{\varepsilon} \psi_{0},{\overline{\left(\mu_{\varepsilon}\right)_{*}^{-1}}}^{-1} \circ \circ \mu_{\varepsilon}\right) d \operatorname{vol}_{g_{\varepsilon}}, \\
& l_{2}=\frac{1}{\varepsilon^{m}} \operatorname{Re} \int_{M}\left(\eta_{\varepsilon} \circ \mu_{\varepsilon}^{-1}\right)\left(\overline{\left(\mu_{\varepsilon}\right)_{*}} \circ\left(\tilde{D}_{g_{\varepsilon}} \psi_{0}-\tilde{D}_{g_{\mathbb{R}^{m}}} \psi_{0}\right) \circ \mu_{\varepsilon}^{-1}, \varphi\right) d \operatorname{vol}_{g} \\
& =\operatorname{Re} \int_{B_{\varepsilon^{-1 / 2}}^{0}} \eta_{\varepsilon} \cdot\left(\tilde{D}_{g_{\varepsilon}} \psi_{0}-\tilde{D}_{g_{\mathbb{R}} m} \psi_{0},{\overline{\left(\mu_{\varepsilon}\right)_{*}}}^{-1} \circ \varphi \circ \mu_{\varepsilon}\right) d \operatorname{vol}_{g_{\varepsilon}}, \\
& l_{3}=\frac{1}{\varepsilon^{m}} \operatorname{Re} \int_{M}\left(\eta_{\varepsilon} \circ \mu_{\varepsilon}^{-1}\right)\left(\overline{\left(\mu_{\varepsilon}\right)_{*}} \circ\left(\tilde{D}_{g_{\mathbb{R}^{m}}} \psi_{0}+a \omega_{\mathbb{C}} g_{\varepsilon} \psi_{0}-b\left|\psi_{0}\right|^{p-2} \psi_{0}\right) \circ \mu_{\varepsilon}^{-1}, \varphi\right) d \operatorname{vol}_{g} \\
& =\operatorname{Re} \int_{B_{\varepsilon^{-1 / 2}}^{0}} \eta_{\varepsilon} \cdot\left(\tilde{D}_{g_{\mathbb{R}}} \psi_{0}+\left(a \circ \mu_{\varepsilon}\right) \omega_{\mathbb{C}} g_{\varepsilon} \psi_{0}-\left(b \circ \mu_{\varepsilon}\right)\left|\psi_{0}\right|^{p-2} \psi_{0}, \overline{\left(\mu_{\varepsilon}\right)_{*}}-1 \circ \varphi \circ \mu_{\varepsilon}\right) d \operatorname{vol}_{g_{\varepsilon}},
\end{aligned}
$$

and

$$
\begin{aligned}
l_{4} & =\frac{1}{\varepsilon^{m}} \operatorname{Re} \int_{M}\left(\overline{\left(\mu_{\varepsilon}\right)_{*}} \circ\left(b \cdot \eta_{\varepsilon}\left|\psi_{0}\right|^{p-2} \psi_{0}-b \cdot \eta_{\varepsilon}^{p-1}\left|\psi_{0}\right|^{p-2} \psi_{0}\right) \circ \mu_{\varepsilon}^{-1}, \varphi\right) d \operatorname{vol}_{g} \\
& =\operatorname{Re} \int_{B_{\varepsilon^{-1 / 2}}^{0}}\left(b \circ \mu_{\varepsilon}\right)\left(\eta_{\varepsilon}-\eta_{\varepsilon}^{p-1}\right)\left|\psi_{0}\right|^{p-2}\left(\psi_{0}, \overline{\left(\mu_{\varepsilon}\right)_{*}^{-1}} \circ \varphi \circ \mu_{\varepsilon}\right) d \operatorname{vol}_{g_{\varepsilon}} .
\end{aligned}
$$

For $l_{1}$, by the Hölder inequality and Lemma 2.4, we have

$$
\begin{aligned}
\left|l_{1}\right| & \leq\left(\int_{B_{\varepsilon^{-1 / 2}}^{0}}\left|\nabla \eta_{\varepsilon} g_{\varepsilon} \psi_{0}\right|^{2} d \operatorname{vol}_{g_{\varepsilon}}\right)^{\frac{1}{2}}\left(\frac{1}{\varepsilon^{m}} \int_{B_{\varepsilon^{-1 / 2}}^{0}}\left|\varphi \circ \mu_{\varepsilon}\right|^{2} d \operatorname{vol}_{\mu_{\varepsilon}^{*} g}\right)^{\frac{1}{2}} \\
& \leq C \varepsilon^{\frac{1}{2}}\left(\int_{B_{\varepsilon^{-1 / 2}}^{0} \backslash B_{\frac{1}{2} \varepsilon^{-1 / 2}}^{0}}\left|\psi_{0}\right|^{2} d \operatorname{vol}_{g_{\mathbb{R}^{m}}}\right)^{\frac{1}{2}} \cdot|\varphi|_{2, \varepsilon} \\
& \leq C \varepsilon^{\frac{1}{2}}\left|\psi_{0}\right|_{2} \cdot\|\varphi\|_{\varepsilon}
\end{aligned}
$$


where we used $d \operatorname{vol}_{g_{\varepsilon}} \leq C d \operatorname{vol}_{g_{\mathbb{R}^{m}}}$ on $B_{\varepsilon^{-1 / 2}}^{0}$ for some constant $C>0$ as $\varepsilon \rightarrow 0$. We soon obtain

$$
\left|l_{1}\right| \leq o_{\varepsilon}(1)\|\varphi\|_{\varepsilon} \quad \text { as } \varepsilon \rightarrow 0 \text {. }
$$

In order to estimate $l_{2}$, let us mention that, by (5.2) and the $L^{p}$-theory for Dirac operators, we have $\nabla \psi_{0} \in L^{\frac{p}{p-1}}\left(\mathbb{R}^{m}, \widetilde{\mathbb{S}}\left(\mathbb{R}^{m}\right)\right)$. And hence, we get

$$
\begin{aligned}
\left|l_{2}\right| & \leq\left(\int_{B_{\varepsilon^{-1 / 2}}^{0}}\left|\tilde{D}_{g_{\varepsilon}} \psi_{0}-\tilde{D}_{g_{\mathbb{R}^{m}}} \psi_{0}\right|^{\frac{p}{p-1}} d \operatorname{vol}_{g_{\varepsilon}}\right)^{\frac{p-1}{p}}\left(\frac{1}{\varepsilon^{m}} \int_{B_{\varepsilon^{-1 / 2}}^{0}}\left|\varphi \circ \mu_{\varepsilon}\right|^{p} d \operatorname{vol}_{\mu_{\varepsilon}^{*} g}\right)^{\frac{1}{p}} \\
& \leq C\left(\int_{B_{\varepsilon^{-1 / 2}}^{0}}\left|\tilde{D}_{g_{\varepsilon}} \psi_{0}-\tilde{D}_{g_{\mathbb{R}}} \psi_{0}\right|^{\frac{p}{p-1}} d \operatorname{vol}_{g_{\mathbb{R}} m}\right)^{\frac{p-1}{p}}\|\varphi\|_{\varepsilon} .
\end{aligned}
$$

Since $g_{\varepsilon} \rightarrow g_{\mathbb{R}^{m}}$ on $B_{\varepsilon^{-1 / 2}}^{0}$ in $C^{\infty}$-topology as $\varepsilon \rightarrow 0$, we can get further from the above estimate that

$$
\left|l_{2}\right| \leq o_{\varepsilon}(1)\|\varphi\|_{\varepsilon} \quad \text { as } \varepsilon \rightarrow 0
$$

The estimate for $l_{3}$ is much more clear. Indeed, by the definition of $\mu_{\varepsilon}$, we have

$$
a \circ \mu_{\varepsilon} \rightarrow \nu_{0} \quad \text { and } \quad b \circ \mu_{\varepsilon} \rightarrow \kappa_{0}
$$

uniformly on $B_{\varepsilon^{-1 / 2}}^{0}$ and, therefore, it follows that

$$
\begin{aligned}
\left|l_{3}\right| \leq & C\left(\int_{B_{\varepsilon^{-1 / 2}}^{0}}\left|a \circ \mu_{\varepsilon}-\nu_{0}\right|^{2}\left|\psi_{0}\right|^{2} d \operatorname{vol}_{\mathbb{R}^{m}}\right)^{\frac{1}{2}}|\varphi|_{2, \varepsilon} \\
& +C\left(\int_{B_{\varepsilon^{0}}^{0} / 2}\left|b \circ \mu_{\varepsilon}-\kappa_{0}\right|^{\frac{p}{p-1}}\left|\psi_{0}\right|^{p} d \operatorname{vol}_{g_{\mathbb{R}^{m}}}\right)^{\frac{p-1}{p}}|\varphi|_{p, \varepsilon} \\
\leq & o_{\varepsilon}(1)\|\varphi\|_{\varepsilon}
\end{aligned}
$$

as $\varepsilon \rightarrow 0$.

It remains to estimate $l_{4}$. Similarly as was argued in the above, we have

$$
\begin{aligned}
\left|l_{4}\right| & \leq C\left(\int_{B_{\varepsilon^{-1 / 2}}^{0}}\left(\eta_{\varepsilon}-\eta_{\varepsilon}^{p-1}\right)^{\frac{p}{p-1}}\left|\psi_{0}\right|^{p} d \operatorname{vol}_{g_{\mathbb{R}} m}\right)^{\frac{p-1}{p}}|\varphi|_{p, \varepsilon} \\
& \leq C\left(\int_{B_{\varepsilon^{-1 / 2}}^{0} \backslash B_{\frac{1}{2} \varepsilon^{-1 / 2}}^{0}}\left|\psi_{0}\right|^{p} d \operatorname{vol}_{g_{\mathbb{R}^{m}}}\right)^{\frac{p-1}{p}}\|\varphi\|_{\varepsilon}
\end{aligned}
$$

where

$$
\int_{B_{\varepsilon^{-1 / 2}}^{0} \backslash B_{\frac{1}{2} \varepsilon^{-1 / 2}}^{0}}\left|\psi_{0}\right|^{p} d \operatorname{vol}_{g_{\mathbb{R}^{m}}} \rightarrow 0
$$

as $\varepsilon \rightarrow 0$. Hence we have

$$
\left|l_{4}\right| \leq o_{\varepsilon}(1)\|\varphi\|_{\varepsilon} \quad \text { as } \varepsilon \rightarrow 0 .
$$

Combining (5.11)-(5.14), we have $\left\|\mathcal{L}_{\varepsilon}^{\prime}\left(\phi_{\varepsilon}\right)\right\|_{\varepsilon} \rightarrow 0$ as $\varepsilon \rightarrow 0$ as desired.

Lemma 5.2. $\mathcal{L}_{\varepsilon}\left(\phi_{\varepsilon}\right) \rightarrow \gamma\left(\nu_{0}, \kappa_{0}\right)$ as $\varepsilon \rightarrow 0$. 
Proof. Due to the definition of the test spinor $\phi_{\varepsilon}$ in (5.4), we can find a constant $C>0$ independent of $\varepsilon$ such that $\left\|\phi_{\varepsilon}\right\|_{\varepsilon} \leq C$. And thus, by Lemma 5.1, we easily see that

$$
\mathcal{L}_{\varepsilon}\left(\phi_{\varepsilon}\right)=\mathcal{L}_{\varepsilon}\left(\phi_{\varepsilon}\right)-\frac{1}{2} \mathcal{L}_{\varepsilon}^{\prime}\left(\phi_{\varepsilon}\right)\left[\phi_{\varepsilon}\right]+o_{\varepsilon}(1)=\frac{p-2}{2 \varepsilon^{m} p} \int_{M} b\left|\phi_{\varepsilon}\right|^{p} d \operatorname{vol}_{g}+o_{\varepsilon}(1)
$$

as $\varepsilon \rightarrow 0$.

Observe that

$$
\frac{1}{\varepsilon^{m}} \int_{M} b\left|\phi_{\varepsilon}\right|^{p} d \operatorname{vol}_{g}=\frac{1}{\varepsilon^{m}} \int_{B_{\varepsilon^{1 / 2}}\left(\xi_{0}\right)} b\left|\phi_{\varepsilon}\right|^{p} d \operatorname{vol}_{g}=\int_{B_{\varepsilon^{-1 / 2}}^{0}} b \circ \mu_{\varepsilon}\left|\eta_{\varepsilon} \cdot \psi_{0}\right|^{p} d \operatorname{vol}_{g_{\varepsilon}},
$$

where

$$
\int_{B_{\varepsilon^{-1 / 2}}^{0}} b \circ \mu_{\varepsilon}\left|\eta_{\varepsilon} \cdot \psi_{0}\right|^{p} d \operatorname{vol}_{g_{\varepsilon}}=\kappa_{0} \int_{\mathbb{R}^{m}}\left|\psi_{0}\right|^{p} d \operatorname{vol}_{g_{\mathbb{R}^{m}}}+o_{\varepsilon}(1)
$$

as $\varepsilon \rightarrow 0$.

Thus, by (5.2) and (5.3), we soon have

$$
\mathcal{L}_{\varepsilon}\left(\phi_{\varepsilon}\right)=\frac{p-2}{2 p} \kappa_{0} \int_{\mathbb{R}^{m}}\left|\psi_{0}\right|^{p} d \operatorname{vol}_{g_{\mathbb{R}^{m}}}+o_{\varepsilon}(1)=\gamma\left(\nu_{0}, \kappa_{0}\right)+o_{\varepsilon}(1)
$$

as $\varepsilon \rightarrow 0$ which completes the proof.

Here, we emphasize that the above two lemmas yield a description of the limiting behavior of the critical value $\gamma_{\varepsilon}$ obtained in (3.3). Namely, applying Corollary 3.6 for $\left\{\phi_{\varepsilon}\right\}$, we obtain the following estimate.

Corollary 5.3. $\limsup _{\varepsilon \rightarrow 0} \gamma_{\varepsilon} \leq \gamma\left(\nu_{0}, \kappa_{0}\right)=\alpha_{\min } \gamma(1,1)$.

\section{Asymptotic profiles of the solutions}

In the present section we will firstly establish a complete description for the model problem (2.7). After that we will return to the analysis of the limiting behavior of the conformal metrics induced by (2.6).

To begin with, let $\left\{\psi_{\varepsilon}\right\}$ be a family of solutions to (2.7) found by (3.3), i.e.,

$$
\mathcal{L}_{\varepsilon}\left(\psi_{\varepsilon}\right)=\gamma_{\varepsilon} \quad \text { and } \quad \mathcal{L}_{\varepsilon}^{\prime}\left(\psi_{\varepsilon}\right)=0
$$

Then, as was mentioned in (3.4), we find

$$
\frac{p-2}{2 \varepsilon^{m} p} \int_{M} b\left|\psi_{\varepsilon}\right|^{p} d \operatorname{vol}_{g}=\gamma_{\varepsilon} \geq \tau_{0}>0
$$

for some $\tau_{0}>0$. In what follows, for any $\xi \in M$ and $r>0, B_{r}(\xi) \subset M$ denote the distance ball of radius $r$ with respect to the metric $g$.

Lemma 6.1. There exist $\xi_{\varepsilon} \in M, r_{0}, \delta_{0}>0$ such that

$$
\liminf _{\varepsilon \rightarrow 0} \frac{1}{\varepsilon^{m}} \int_{B_{\varepsilon r_{0}}\left(\xi_{\varepsilon}\right)}\left|\psi_{\varepsilon}\right|^{2} d \operatorname{vol}_{g} \geq \delta_{0} .
$$


Proof. Assume on the contrary that for any $r>0$

$$
\sup _{\xi \in M} \frac{1}{\varepsilon^{m}} \int_{B_{2 \varepsilon r}(\xi)}\left|\psi_{\varepsilon}\right|^{2} d \operatorname{vol}_{g} \rightarrow 0 \quad \text { as } \varepsilon \rightarrow 0 .
$$

For each $\xi \in M$, let us now choose a smooth real cut-off function $\chi_{\xi, \varepsilon} \equiv 1$ on $B_{\varepsilon r}(\xi)$ and $\operatorname{supp} \chi_{\xi, \varepsilon} \subset B_{2 \varepsilon r}(\xi)$. Then, for $s \in(0,1)$, we consider $q_{s}=2+\left(m^{*}-2\right) s \in\left(2, m^{*}\right)$ and we have

$$
\int_{B_{2 \varepsilon r}(\xi)}\left|\chi_{\xi, \varepsilon} \psi_{\varepsilon}\right|^{q_{s}} d \operatorname{vol}_{g} \leq\left(\int_{B_{2 \varepsilon r}(\xi)}\left|\chi_{\xi, \varepsilon} \psi_{\varepsilon}\right|^{2} d \operatorname{vol}_{g}\right)^{1-s}\left(\int_{B_{2 \varepsilon r}(\xi)}\left|\chi_{\xi, \varepsilon} \psi_{\varepsilon}\right|^{\frac{2 m}{m-1}} d \operatorname{vol}_{g}\right)^{s} .
$$

Taking $s=\frac{2}{m^{*}}$, we obtain from Lemma 2.4 that

$$
\left(\frac{1}{\varepsilon^{m}} \int_{B_{2 \varepsilon r}(\xi)}\left|\chi_{\xi, \varepsilon} \psi_{\varepsilon}\right|^{m^{*}} d \operatorname{vol}_{g}\right)^{s} \leq C\left\|\chi_{\xi, \varepsilon} \psi_{\varepsilon}\right\|_{\varepsilon}^{2}
$$

Now, covering $M$ by balls of radius $\varepsilon r$ such that any point $\xi \in M$ is contained in at most $K_{M}$ balls, where $K_{M}$ does not depend on $\varepsilon$. This condition can be satisfied for $\varepsilon$ small by the compactness of $M$. And thus, we find

$$
\frac{1}{\varepsilon^{m}} \int_{M}\left|\psi_{\varepsilon}\right|^{q_{s}} d \operatorname{vol}_{g} \leq C \cdot K_{M}\left(\sup _{\xi \in M} \int_{B_{2 \varepsilon r}(\xi)}\left|\chi_{\xi, \varepsilon} \psi_{\varepsilon}\right|^{2} d \operatorname{vol}_{g}\right)^{1-s}\left\|\psi_{\varepsilon}\right\|_{\varepsilon}^{2}
$$

Notice that $\left\|\psi_{\varepsilon}\right\|_{\varepsilon}$ is bounded, it follows from (6.3) that $\left|\psi_{\varepsilon}\right|_{q_{s}, \varepsilon} \rightarrow 0$. Since $2<q_{s}<m^{*}$, we see easily that $\left|\psi_{\varepsilon}\right|_{q, \varepsilon} \rightarrow 0$ for all $q \in\left(2, m^{*}\right)$ which contradict to (6.2)

We may now fix the sequence $\left\{\xi_{\varepsilon}\right\} \subset M$ and the constants $r_{0}, \delta_{0}>0$ in Lemma 6.1. Up to a subsequence if necessary, we assume that $\xi_{\varepsilon} \rightarrow \xi_{\infty} \in M$ as $\varepsilon \rightarrow 0$. Then, similar to Section 5. we can consider the rescaled geodesic normal coordinates near each $\xi_{\varepsilon}$ via the formula

$$
\Theta_{\varepsilon}(x)=\exp _{\xi_{\varepsilon}}(\varepsilon x)
$$

For any $R \geq r_{0}$, we have a conformal equivalence $\left(B_{R}^{0}, \varepsilon^{-2} \Theta_{\varepsilon}^{*} g\right) \cong\left(B_{\varepsilon R}\left(\xi_{\varepsilon}\right), g\right)$ for all small $\varepsilon$. Denoting by $g_{\Theta_{\varepsilon}}=\varepsilon^{-2} \Theta_{\varepsilon}^{*} g$, then we have $g_{\Theta_{\varepsilon}} \rightarrow g_{\mathbb{R}^{m}}$ in $C^{\infty}\left(B_{R}^{0}\right)$ as $\varepsilon \rightarrow 0$.

Let $\overline{\left(\Theta_{\varepsilon}\right)_{*}}: \mathbb{S}_{x}\left(B_{R}^{0}, g_{\Theta_{\varepsilon}}\right) \rightarrow \mathbb{S}_{\Theta_{\varepsilon}(x)}\left(B_{\varepsilon R}\left(\xi_{\varepsilon}\right), g\right)$ denote the bundle identification for spinors, we can introduce a family of spinors on $B_{R}^{0}$ by

$$
z_{\varepsilon}={\overline{\left(\Theta_{\varepsilon}\right)_{*}}}^{-1} \circ \psi_{\varepsilon} \circ \Theta_{\varepsilon}
$$

Along this line of consideration, we have

$$
\begin{gathered}
\tilde{D}_{g_{\Theta_{\varepsilon}}} z_{\varepsilon}=\overline{\left(\Theta_{\varepsilon}\right)_{*}^{-1}} \circ\left(\varepsilon \tilde{D}_{g} \psi_{\varepsilon}\right) \circ \Theta_{\varepsilon}, \\
\int_{B_{R}^{0}}\left(\tilde{D}_{g_{\Theta_{\varepsilon}}} z_{\varepsilon}, z_{\varepsilon}\right) d \operatorname{vol}_{\Theta_{\Theta_{\varepsilon}}}=\frac{1}{\varepsilon^{m}} \int_{B_{\varepsilon R}\left(\xi_{\varepsilon}\right)}\left(\varepsilon \tilde{D}_{g} \psi_{\varepsilon}, \psi_{\varepsilon}\right) d \operatorname{vol}_{g}, \\
\int_{B_{R}^{0}}\left(\omega_{\mathbb{C}} g_{g_{\Theta_{\varepsilon}}} z_{\varepsilon}, z_{\varepsilon}\right) d \operatorname{vol}_{g_{\Theta_{\varepsilon}}}=\frac{1}{\varepsilon^{m}} \int_{B_{\varepsilon R}\left(\xi_{\varepsilon}\right)}\left(\omega_{\mathbb{C} \cdot g} \psi_{\varepsilon}, \psi_{\varepsilon}\right) d \operatorname{vol}_{g},
\end{gathered}
$$




$$
\int_{B_{R}^{0}}\left|z_{\varepsilon}\right|^{p} d \operatorname{vol}_{g_{\Theta_{\varepsilon}}}=\frac{1}{\varepsilon^{m}} \int_{B_{\varepsilon R}\left(\xi_{\varepsilon}\right)}\left|\psi_{\varepsilon}\right|^{p} d \operatorname{vol}_{g} .
$$

Particularly, since $\left\|\psi_{\varepsilon}\right\|_{\varepsilon}$ is bounded, we have

$$
0<\liminf _{\varepsilon \rightarrow 0} \int_{B_{R}^{0}}\left|z_{\varepsilon}\right|^{p} d \operatorname{vol}_{g_{\Theta_{\varepsilon}}} \leq \limsup _{\varepsilon \rightarrow 0} \frac{1}{\varepsilon^{m}} \int_{M}\left|\psi_{\varepsilon}\right|^{p} d \operatorname{vol}_{g}<\infty
$$

for any $R>r_{0}$.

Recalling the set $\mathcal{C} \subset M$ defined in (5.1), we have

Lemma 6.2. $\xi_{\infty} \in \mathcal{C}$, i.e., $\operatorname{dist}_{g}\left(\xi_{\varepsilon}, \mathcal{C}\right) \rightarrow 0$ as $\varepsilon \rightarrow 0$.

Proof. Since $\left\{z_{\varepsilon}\right\}$ is $W_{\text {loc }}^{\frac{1}{2}, 2}\left(\mathbb{R}^{m}, \tilde{\mathbb{S}}\left(\mathbb{R}^{m}\right)\right)$-bounded, that is, $\left\{\beta z_{\varepsilon}\right\} \subset W^{\frac{1}{2}, 2}\left(\mathbb{R}^{m}, \widetilde{\mathbb{S}}\left(\mathbb{R}^{m}\right)\right)$ is bounded for any $\beta \in C_{c}^{\infty}\left(\mathbb{R}^{m}\right)$. We can assume, up to a subsequence, $z_{\varepsilon} \rightarrow z_{\infty}$ in $W_{l o c}^{\frac{1}{2}, 2}\left(\mathbb{R}^{m}, \tilde{\mathbb{S}}\left(\mathbb{R}^{m}\right)\right)$ and $z_{\varepsilon} \rightarrow z_{\infty}$ in $L_{\text {loc }}^{q}\left(\mathbb{R}^{m}, \tilde{\mathbb{S}}\left(\mathbb{R}^{m}\right)\right)$ for $2 \leq q<\frac{2 m}{m-1}$. At the same time, by (6.8), we see easily that $z_{\infty} \in L^{p}\left(\mathbb{R}^{m}, \tilde{\mathbb{S}}\left(\mathbb{R}^{m}\right)\right)$.

Let $\varphi \in W^{\frac{1}{2}, 2}\left(\mathbb{R}^{m}, \widetilde{\mathbb{S}}\left(\mathbb{R}^{m}\right)\right)$ be such that $\operatorname{supp} \varphi$ is compact, i.e. $\operatorname{supp} \varphi \subset B_{R}^{0}$ for some $R$ large. Then, we have

$$
\begin{aligned}
\int_{\mathbb{R}^{m}} & \left(\tilde{D}_{g_{\mathbb{R}^{m}}} z_{\infty}+a\left(\xi_{\infty}\right) \omega_{\mathbb{C}} \cdot g_{\mathbb{R}^{m}} z_{\infty}-b\left(\xi_{\infty}\right)\left|z_{\infty}\right|^{p-2} z_{\infty}, \varphi\right) d \operatorname{vol}_{g_{\mathbb{R}^{m}}} \\
& =\lim _{\varepsilon \rightarrow 0} \int_{\operatorname{supp} \varphi}\left(\tilde{D}_{g_{\Theta_{\varepsilon}}} z_{\varepsilon}+\left(a \circ \Theta_{\varepsilon}\right) \omega_{\mathbb{C}} g_{g_{\Theta}} z_{\varepsilon}-\left(b \circ \Theta_{\varepsilon}\right)\left|z_{\varepsilon}\right|^{p-2} z_{\varepsilon}, \varphi\right) d \operatorname{vol}_{g_{\Theta_{\varepsilon}}} \\
& =\lim _{\varepsilon \rightarrow 0} \frac{1}{\varepsilon^{m}} \int_{B_{\varepsilon R}\left(\xi_{\varepsilon}\right)}\left(\varepsilon \tilde{D}_{g} \psi_{\varepsilon}+a \omega_{\mathbb{C} \cdot g} \psi_{\varepsilon}-b\left|\psi_{\varepsilon}\right|^{p-2} \psi_{\varepsilon}, \overline{\left(\Theta_{\varepsilon}\right)_{*}} \circ \varphi \circ \Theta_{\varepsilon}^{-1}\right) d \operatorname{vol}_{g} \\
& =0
\end{aligned}
$$

Hence, we have $z_{\infty}$ satisfies

$$
\tilde{D}_{g_{\mathbb{R}^{m}}} z_{\infty}+a\left(\xi_{\infty}\right) \omega_{\mathbb{C}} \cdot g_{\mathbb{R}^{m}} z_{\infty}=b\left(\xi_{\infty}\right)\left|z_{\infty}\right|^{p-2} z_{\infty} \quad \text { on } \mathbb{R}^{m}
$$

This implies, by the elliptic regularity, $\tilde{D}_{g_{\mathbb{R}} m} z_{\infty}+a\left(\xi_{\infty}\right) \omega_{\mathbb{C}} \cdot g_{\mathbb{R}^{m}} z_{\infty} \in L^{\frac{p}{p-1}}\left(\mathbb{R}^{m}, \widetilde{\mathbb{S}}\left(\mathbb{R}^{m}\right)\right)$. Moreover, combined with the Sobolev embedding $L^{\frac{p}{p-1}}\left(\mathbb{R}^{m}, \tilde{\mathbb{S}}\left(\mathbb{R}^{m}\right)\right) \hookrightarrow W^{-\frac{1}{2}, 2}\left(\mathbb{R}^{m}, \tilde{\mathbb{S}}\left(\mathbb{R}^{m}\right)\right)$, we get $z_{\infty} \in W^{\frac{1}{2}, 2}\left(\mathbb{R}^{m}, \widetilde{\mathbb{S}}\left(\mathbb{R}^{m}\right)\right)$.

Now, by collecting (6.1), (6.2) and (6.5)-(6.8), we conclude that $z_{\infty}$ is a nontrivial solution to (6.9) and

$$
\begin{aligned}
\limsup _{\varepsilon \rightarrow 0} \mathcal{L}_{\varepsilon}\left(\psi_{\varepsilon}\right) & =\limsup _{\varepsilon \rightarrow 0} \frac{p-2}{2 p \varepsilon^{m}} \int_{M} b\left|\psi_{\varepsilon}\right|^{p} d \operatorname{vol}_{g} \\
& \geq \liminf _{\varepsilon \rightarrow 0} \frac{p-2}{2 p} b\left(\xi_{\infty}\right) \int_{B_{R}^{0}}\left|z_{\varepsilon}\right|^{p} d \operatorname{vol}_{\Theta_{\Theta_{\varepsilon}}} \\
& \geq \frac{p-2}{2 p} b\left(\xi_{\infty}\right) \int_{B_{R}^{0}}\left|z_{\infty}\right|^{p} d \operatorname{vol}_{\mathbb{R}^{m}}
\end{aligned}
$$

where in the last inequality we have used the Fatou's lemma. Due to the arbitrariness of $R>0$, combined with the results obtained in Section 4, we have

$$
\limsup _{\varepsilon \rightarrow 0} \mathcal{L}_{\varepsilon}\left(\psi_{\varepsilon}\right) \geq \gamma\left(a\left(\xi_{\infty}\right), b\left(\xi_{\infty}\right)\right)=\alpha\left(\xi_{\infty}\right) \gamma(1,1)
$$

Then Corollary 5.3 suggests $\alpha\left(\xi_{\infty}\right)=\alpha_{\text {min }}$, i.e., $\xi_{\infty} \in \mathcal{C}$, which completes the proof. 
With Lemma 6.1 and 6.2 in hand, we may now choose $\beta \in C^{\infty}(M)$ be a cut-off function such that $\beta \equiv 1$ on $B_{r}\left(\xi_{\infty}\right)$ and $\operatorname{supp} \beta \subset B_{2 r}\left(\xi_{\infty}\right)$ for some $r>0$ and define spinors on $M$ as

$$
\zeta_{\varepsilon}=\beta(\cdot) \overline{\left(\Theta_{\varepsilon}\right)_{*}} \circ z_{\infty} \circ \Theta_{\varepsilon}^{-1}
$$

Setting $w_{\varepsilon}=\psi_{\varepsilon}-\zeta_{\varepsilon}$, we soon have $\left|w_{\varepsilon}\right|_{p, \varepsilon} \rightarrow 0$ as $\varepsilon \rightarrow 0$ (otherwise, we can apply Lemma 6.1 and 6.2 for $\left\{w_{\varepsilon}\right\}$ instead of $\left\{\psi_{\varepsilon}\right\}$ to get $\mathcal{L}_{\varepsilon}\left(\psi_{\varepsilon}\right) \geq 2 \alpha_{\min } \gamma(1,1)$ which is absurd).

Lemma 6.3. $\left\|\mathcal{L}_{\varepsilon}^{\prime}\left(\zeta_{\varepsilon}\right)\right\|_{\varepsilon} \rightarrow 0$ and $\left\|\mathcal{L}_{\varepsilon}^{\prime}\left(w_{\varepsilon}\right)\right\|_{\varepsilon} \rightarrow 0$ as $\varepsilon \rightarrow 0$.

Proof. We point out that, after some minor revision, the proof of Lemma 5.1 can be applied here to show $\left\|\mathcal{L}_{\varepsilon}^{\prime}\left(\zeta_{\varepsilon}\right)\right\|_{\varepsilon} \rightarrow 0$ as $\varepsilon \rightarrow 0$. Hence, we only need to check the second estimate.

Again, we choose $\varphi \in \mathcal{H}$ be an arbitrary test spinor. We then have

$$
\begin{aligned}
\mathcal{L}_{\varepsilon}^{\prime}\left(w_{\varepsilon}\right)[\varphi] & =\frac{1}{\varepsilon^{m}} \operatorname{Re} \int_{M}\left(\varepsilon \tilde{D}_{g} w_{\varepsilon}+a \omega_{\mathbb{C}} \cdot w_{\varepsilon}-b\left|w_{\varepsilon}\right|^{p-2} w_{\varepsilon}, \varphi\right) d \operatorname{vol}_{g} \\
& =\mathcal{L}_{\varepsilon}^{\prime}\left(\psi_{\varepsilon}\right)[\varphi]-\mathcal{L}_{\varepsilon}^{\prime}\left(\zeta_{\varepsilon}\right)[\varphi]+\frac{1}{\varepsilon^{m}} \operatorname{Re} \int_{M}\left(\Psi_{\varepsilon}, \varphi\right) d \operatorname{vol}_{g},
\end{aligned}
$$

where

$$
\Psi_{\varepsilon}=b\left|\psi_{\varepsilon}\right|^{p-2} \psi_{\varepsilon}-b\left|\zeta_{\varepsilon}\right|^{p-2} \zeta_{\varepsilon}-b\left|w_{\varepsilon}\right|^{p-2} w_{\varepsilon}
$$

Since $\mathcal{L}_{\varepsilon}^{\prime}\left(\psi_{\varepsilon}\right)=0$ for all $\varepsilon$ small, it remains to estimate the last integral in (6.10).

To proceed, we first mention that there exists $C>0$ (independent of $\varepsilon$ ) such that

$$
\left|\Psi_{\varepsilon}\right| \leq C\left|\zeta_{\varepsilon}\right|^{p-2}\left|w_{\varepsilon}\right|+C\left|w_{\varepsilon}\right|^{p-2}\left|\zeta_{\varepsilon}\right|
$$

Thus, for any $R>0$, we have

$$
\begin{aligned}
& \frac{1}{\varepsilon^{m}} \int_{M \backslash B_{\varepsilon R}\left(\xi_{\varepsilon}\right)}\left|\zeta_{\varepsilon}\right|^{p-2} \cdot\left|w_{\varepsilon}\right| \cdot|\varphi| d \operatorname{vol}_{g} \\
& \quad \leq\left(\frac{1}{\varepsilon^{m}} \int_{M \backslash B_{\varepsilon R}\left(\xi_{\varepsilon}\right)}\left|\zeta_{\varepsilon}\right|^{p} d \operatorname{vol}_{g}\right)^{\frac{p-2}{p^{*}}}\left(\frac{1}{\varepsilon^{m}} \int_{M \backslash B_{\varepsilon R}\left(\xi_{\varepsilon}\right)}\left|w_{\varepsilon}\right|^{p} d \operatorname{vol}_{g}\right)^{\frac{1}{p}}|\varphi|_{p, \varepsilon} \\
& \quad \leq C\left(\int_{B_{2 r / \varepsilon}^{0} \backslash B_{R}^{0}}\left|z_{\infty}\right|^{p} d \operatorname{vol}_{g_{\Theta_{\varepsilon}}}\right)^{\frac{p-2}{p}}\left\|w_{\varepsilon}\right\|_{\varepsilon} \cdot\|\varphi\|_{\varepsilon}=o_{R}(1)\|\varphi\|_{\varepsilon}
\end{aligned}
$$

and

$$
\begin{aligned}
& \frac{1}{\varepsilon^{m}} \int_{M \backslash B_{\varepsilon R}\left(\xi_{\varepsilon}\right)}\left|w_{\varepsilon}\right|^{p-2} \cdot\left|\zeta_{\varepsilon}\right| \cdot|\varphi| d \operatorname{vol}_{g} \\
& \quad \leq\left(\frac{1}{\varepsilon^{m}} \int_{M \backslash B_{\varepsilon R}\left(\xi_{\varepsilon}\right)}\left|w_{\varepsilon}\right|^{p} d \operatorname{vol}_{g}\right)^{\frac{p-2}{p}}\left(\frac{1}{\varepsilon^{m}} \int_{M \backslash B_{\varepsilon R}\left(\xi_{\varepsilon}\right)}\left|\zeta_{\varepsilon}\right|^{p} d \operatorname{vol}_{g}\right)^{\frac{1}{p}}|\varphi|_{p, \varepsilon} \\
& \quad \leq C\left(\int_{B_{2 r / \varepsilon}^{0} \backslash B_{R}^{0}}\left|z_{\infty}\right|^{p} d \operatorname{vol}_{g_{\Theta \varepsilon}}\right)^{\frac{1}{p}}\left\|w_{\varepsilon}\right\|_{\varepsilon}^{p-2} \cdot\|\varphi\|_{\varepsilon}=o_{R}(1)\|\varphi\|_{\varepsilon},
\end{aligned}
$$


where $o_{R}(1) \rightarrow 0$ as $R \rightarrow \infty$. At the same time, inside $B_{\varepsilon R}\left(\xi_{\varepsilon}\right)$, we have

$$
\begin{aligned}
& \frac{1}{\varepsilon^{m}} \int_{B_{\varepsilon R}\left(\xi_{\varepsilon}\right)}\left|\zeta_{\varepsilon}\right|^{p-2} \cdot\left|w_{\varepsilon}\right| \cdot|\varphi| d \operatorname{vol}_{g} \\
& \quad \leq\left(\frac{1}{\varepsilon^{m}} \int_{B_{\varepsilon R}\left(\xi_{\varepsilon}\right)}\left|\zeta_{\varepsilon}\right|^{p} d \operatorname{vol}_{g}\right)^{\frac{p-2}{p}}\left(\frac{1}{\varepsilon^{m}} \int_{B_{\varepsilon R}\left(\xi_{\varepsilon}\right)}\left|w_{\varepsilon}\right|^{p} d \operatorname{vol}_{g}\right)^{\frac{1}{p}}|\varphi|_{p, \varepsilon} \\
& \quad \leq C\left(\int_{\mathbb{R}^{m}}\left|z_{\infty}\right|^{p} d \operatorname{vol}_{g_{\mathbb{R}^{m}}}\right)^{\frac{p-2}{p}}\left(\int_{B_{R}^{0}}\left|z_{\varepsilon}-z_{\infty}\right|^{p} d \operatorname{vol}_{g_{\mathbb{R}^{m}}}\right)^{\frac{1}{p}} \cdot\|\varphi\|_{\varepsilon}=o_{\varepsilon}(1)\|\varphi\|_{\varepsilon}
\end{aligned}
$$

and

$$
\begin{aligned}
& \frac{1}{\varepsilon^{m}} \int_{B_{\varepsilon R}\left(\xi_{\varepsilon}\right)}\left|w_{\varepsilon}\right|^{p-2} \cdot\left|\zeta_{\varepsilon}\right| \cdot|\varphi| d \operatorname{vol}_{g} \\
& \quad \leq\left(\frac{1}{\varepsilon^{m}} \int_{B_{\varepsilon R}\left(\xi_{\varepsilon}\right)}\left|w_{\varepsilon}\right|^{p} d \operatorname{vol}_{g}\right)^{\frac{p-2}{p}}\left(\frac{1}{\varepsilon^{m}} \int_{B_{\varepsilon R}\left(\xi_{\varepsilon}\right)}\left|\zeta_{\varepsilon}\right|^{p} d \operatorname{vol}_{g}\right)^{\frac{1}{p}}|\varphi|_{p, \varepsilon} \\
& \quad \leq C\left(\int_{B_{R}^{0}}\left|z_{\varepsilon}-z_{\infty}\right|^{p} d \operatorname{vol}_{g_{\mathbb{R}^{m}}}\right)^{\frac{p-2}{p}}\left(\int_{\mathbb{R}^{m}}\left|z_{\infty}\right|^{p} d \operatorname{vol}_{g_{\mathbb{R}^{m}}}\right)^{\frac{1}{p}} \cdot\|\varphi\|_{\varepsilon}=o_{\varepsilon}(1)\|\varphi\|_{\varepsilon}
\end{aligned}
$$

as $\varepsilon \rightarrow 0$, where we have used $z_{\varepsilon} \rightarrow z_{\infty}$ in $W_{l o c}^{\frac{1}{2}, 2}\left(\mathbb{R}^{m}, \tilde{\mathbb{S}}\left(\mathbb{R}^{m}\right)\right)$ and the compact Sobolev embedding $W_{l o c}^{\frac{1}{2}, 2}\left(\mathbb{R}^{m}, \tilde{\mathbb{S}}\left(\mathbb{R}^{m}\right)\right) \hookrightarrow L_{l o c}^{p}\left(\mathbb{R}^{m}, \tilde{\mathbb{S}}\left(\mathbb{R}^{m}\right)\right)$.

Therefore, we can conclude that

$$
\frac{1}{\varepsilon^{m}} \operatorname{Re} \int_{M}\left(\Psi_{\varepsilon}, \varphi\right) d \operatorname{vol}_{g}=o_{\varepsilon}(1)\|\varphi\|_{\varepsilon} \quad \text { as } \varepsilon \rightarrow 0
$$

And hence, by (6.10), we have $\left\|\mathcal{L}_{\varepsilon}^{\prime}\left(w_{\varepsilon}\right)\right\|_{\varepsilon} \rightarrow 0$ as $\varepsilon \rightarrow 0$.

At this point, we have the following result which summarizes the concentration phenomenon of the family $\left\{\psi_{\varepsilon}\right\}$ for the model problem (2.7).

Proposition 6.4. Let $\left\{\psi_{\varepsilon}\right\}$ be the family of solutions to (2.7) found by (3.3). Then there exist a convergent sequence $\left\{\xi_{\varepsilon}\right\} \subset M, \xi_{\varepsilon} \rightarrow \xi_{\infty}$ as $\varepsilon \rightarrow 0$ and a non-trivial solution $z_{\infty}$ of Eq. (6.9) such that

$$
\alpha\left(\xi_{\infty}\right)=\alpha_{\min }
$$

and

$$
\psi_{\varepsilon}=\beta\left(\cdot \overline{\left(\Theta_{\varepsilon}\right)_{*}} \circ z_{\infty} \circ \Theta_{\varepsilon}^{-1}+w_{\varepsilon} \quad \text { in } \mathcal{H}\right.
$$

where $\left\|w_{\varepsilon}\right\|_{\varepsilon} \rightarrow 0$ as $\varepsilon \rightarrow 0, \Theta_{\varepsilon}(x)=\exp _{\xi_{\varepsilon}}(\varepsilon x)$ and $\beta \in C^{\infty}(M)$ is a cut-off function such that $\beta \equiv 1$ on $B_{r}\left(\xi_{\infty}\right)$ and $\operatorname{supp} \beta \subset B_{2 r}\left(\xi_{\infty}\right)$, some $r>0$. Moreover, there holds

$$
\lim _{\varepsilon \rightarrow 0} \gamma_{\varepsilon}=\lim _{\varepsilon \rightarrow 0} \mathcal{L}_{\varepsilon}\left(\psi_{\varepsilon}\right)=\alpha_{\min } \gamma(1,1)
$$

Proof. By collecting Lemmas 6.1 6.2, it remains to show that $\left\|w_{\varepsilon}\right\|_{\varepsilon} \rightarrow 0$ as $\varepsilon \rightarrow 0$ in (6.12). 
Since $\left\|\mathcal{L}_{\varepsilon}^{\prime}\left(w_{\varepsilon}\right)\right\|_{\varepsilon} \rightarrow 0$ is already suggested by Lemma 6.3, we soon have

$$
\begin{aligned}
\left\|w_{\varepsilon}\right\|_{\varepsilon} & \leq \frac{1}{\varepsilon^{m}} \int_{M} b\left|w_{\varepsilon}\right|^{p-1}\left|w_{\varepsilon}^{+}-w_{\varepsilon}^{-}\right| d \operatorname{vol}_{g}+o_{\varepsilon}(1) \\
& \leq\left(\frac{1}{\varepsilon^{m}} \int_{M} b\left|w_{\varepsilon}\right|^{p} d \operatorname{vol}_{g}\right)^{\frac{p-1}{p}}\left(\frac{1}{\varepsilon^{m}} \int_{M} b\left|w_{\varepsilon}^{+}-w_{\varepsilon}^{-}\right|^{p} d \operatorname{vol}_{g}\right)^{\frac{1}{p}}+o_{\varepsilon}(1) \\
& \leq C\left|w_{\varepsilon}\right|_{p, \varepsilon}\left\|w_{\varepsilon}\right\|+o_{\varepsilon}(1)
\end{aligned}
$$

as $\varepsilon \rightarrow 0$. As was remarked before Lemma 6.3, we have $\left|w_{\varepsilon}\right|_{p, \varepsilon} \rightarrow 0$. Thus, we can infer $\left\|w_{\varepsilon}\right\|_{\varepsilon} \rightarrow 0$ as $\varepsilon \rightarrow 0$.

The above proposition yields a description of the profiles of the solutions to our original problem (2.6). Namely, we can simply substitute $m=m_{1}, p=n^{*}=\frac{2\left(m_{1}+m_{2}\right)}{m_{1}+m_{2}-1}$

$$
a=\lambda \theta \quad \text { and } \quad b=\theta^{m_{1}-\frac{m_{1}-1}{2} n^{*}}
$$

into Eq. (2.7) and calculate the potential function as

$$
\alpha=a^{-\left(m_{1}-1\right)+\frac{2}{n^{*}-2}} b^{-\frac{2}{n^{*}-2}} \equiv \lambda^{m_{2}} \quad \text { on } M_{1} .
$$

Applying the same argument that we have done previously, we obtain our main result as a corollary of Proposition 6.4] (cf. [4, Chapter 3] for regularity results of Dirac operators).

Theorem 6.5. There exists $\varepsilon_{0}>0$ such that, for any $\varepsilon \in\left(0, \varepsilon_{0}\right)$, Eq. (2.6) has a solution $\psi_{\varepsilon} \in$ $C^{1}\left(M_{1}, \tilde{\mathbb{S}}\left(M_{1}\right)\right) \cap C^{\infty}\left(M_{1} \backslash \psi_{\varepsilon}^{-1}(0), \tilde{\mathbb{S}}\left(M_{1}\right)\right)$. Furthermore, there exist a convergent sequence $\left\{\xi_{\varepsilon}\right\} \subset M_{1}, \xi_{\varepsilon} \rightarrow \xi_{0}$ as $\varepsilon \rightarrow 0$ and a non-trivial solution $z_{0}$ of

$$
\tilde{D}_{g_{\mathbb{R}^{m_{1}}}} z+\lambda \theta\left(\xi_{0}\right) \omega_{\mathbb{C}} \cdot g_{\mathbb{R}^{m_{1}}} z=\theta\left(\xi_{0}\right)^{m_{1}-\frac{m_{1}-1}{2} n^{*}}|z|^{n^{*}-2} z \quad \text { on } \mathbb{R}^{m_{1}}
$$

such that

$$
\psi_{\varepsilon}=\beta(\cdot) \overline{\left(\Theta_{\varepsilon}\right)_{*}} \circ z_{0} \circ \Theta_{\varepsilon}^{-1}+w_{\varepsilon} \text { in } \mathcal{H}
$$

where $\left\|w_{\varepsilon}\right\|_{\varepsilon} \rightarrow 0$ as $\varepsilon \rightarrow 0, \Theta_{\varepsilon}(x)=\exp _{\xi_{\varepsilon}}(\varepsilon x)$ and $\beta \in C^{\infty}\left(M_{1}\right)$ is a cut-off function such that $\beta \equiv 1$ on $B_{r}\left(\xi_{\infty}\right)$ and $\operatorname{supp} \beta \subset B_{2 r}\left(\xi_{\infty}\right)$, some $r>0$. Moreover, there holds

$$
\lim _{\varepsilon \rightarrow 0} \frac{1}{\varepsilon^{m_{1}}} \int_{M_{1}} \theta^{m_{1}-\frac{m_{1}-1}{2} n^{*}}\left|\psi_{\varepsilon}\right|^{n^{*}} d \operatorname{vol}_{g}=2 n \cdot \lambda^{m_{2}} \cdot \gamma(1,1) .
$$

\section{Application: CMC immersions for Unduloids}

In this section, comparing with that we have considered previously, we will consider the simplest case of the product construction.

For a given positive $\ell$, we can choose a parameter $t$ on $S^{1}$ via the identification $S^{1}=$ $\mathbb{R} / 2 \pi \ell \mathbb{Z}$. And from now on, $\ell S^{1}$ will stand for this parametrization. Then spinors on $\ell S^{1}$ can be viewed as complex vector functions on $\mathbb{R}$ which are periodic and such that $2 \pi \ell$ is a period. Particularly, on $N=\ell S^{1} \times S^{1}$, we may write $g=d t^{2} \oplus d \tau^{2}$, where $d \tau^{2}$ is the standard metric on the second factor with total length $2 \pi$. 
As was shown in Section 2.1, one dimension spinor space is simply $\mathbb{C}$ and the Clifford multiplication by positively oriented unit vector is multiplication by $i$. With respect to the coordinates $t$ of $\ell S^{1}$ and $\tau$ of $S^{1}$, the Dirac operator on $M$ can be written as

$$
D_{g} \psi=i\left(\frac{d}{d t} \psi_{1} \oplus-\frac{d}{d t} \psi_{2}\right) \otimes \varphi-\left(\psi_{2} \oplus-\psi_{1}\right) \otimes \frac{d}{d \tau} \varphi
$$

for all $\psi=\left(\psi_{1} \oplus \psi_{2}\right) \otimes \varphi \in \Gamma(\mathbb{S}(N))$ where the spinor bundle of $N$ is

$$
\mathbb{S}(N)=\left(\mathbb{S}\left(\ell S^{1}\right) \oplus \mathbb{S}\left(\ell S^{1}\right)\right) \otimes \mathbb{S}\left(S^{1}\right) \cong \mathbb{C}^{2}
$$

We point out that $S^{1}$ has two different spin structures. Recall that a spin structure is a twofold covering of the frame bundle $P_{S O}\left(S^{1}\right)$. Hence we can either take the trivial covering $\sigma_{1}$ : $S^{1} \times \mathbb{Z}_{2} \rightarrow S^{1}$ given by two copies of the identity or we may take $\sigma_{2}: S^{1} \rightarrow S^{1}$ via the mapping $z \mapsto z^{2}$ in complex notation. Furthermore, it is interesting to see that eigenvalues and eigenspinors can be explicitly computed in both structures since a Fourier decomposition is available in this situation. And particularly, in each spin structure, all the eigenspinors are of constant length. To give an idea of our results, we simply consider the first positive eigenvalue of the Dirac operator $D_{g_{S^{1}}}=i \frac{d}{d \tau}$ on $\left(S^{1}, g_{S^{1}}, \sigma\right)$, i.e.

$$
D_{g_{S^{1}}} \varphi_{S^{1}}=\lambda_{1} \varphi_{S^{1}}
$$

with $\lambda_{1}=1, \varphi_{S^{1}}=e^{-i \tau}$ if $\sigma=\sigma_{1}$ or $\lambda_{1}=\frac{1}{2}, \varphi_{S^{1}}=e^{-i \tau / 2}$ if $\sigma=\sigma_{2}$. The proof remains the same if one considers other eigenvalues.

Substituting $\psi=\left(\psi_{1} \oplus \psi_{2}\right) \otimes \varphi_{S^{1}}$ into the conformal invariant equation

$$
D_{g} \psi=|\psi|^{2} \psi \quad \text { on } N=\ell S^{1} \times S^{1},
$$

we are led to an equivalent system of equations

$$
\left\{\begin{aligned}
i \frac{d}{d t} \psi_{1}+i \lambda_{1} \psi_{2} & =\left(\left|\psi_{1}\right|^{2}+\left|\psi_{2}\right|^{2}\right) \psi_{1} \\
-i \frac{d}{d t} \psi_{2}-i \lambda_{1} \psi_{1} & =\left(\left|\psi_{1}\right|^{2}+\left|\psi_{2}\right|^{2}\right) \psi_{2}
\end{aligned}\right.
$$

where $\psi_{1}, \psi_{2}: \mathbb{R} / 2 \pi \ell \mathbb{Z} \rightarrow \mathbb{C}$.

Notice that we can write $\psi_{1}=u_{1}+i v_{1}$ and $\psi_{2}=u_{2}+i v_{2}$ for real functions $u_{1}, u_{2}, v_{1}, v_{2}$. And moreover, Eq. (7.2) is invariant under the multiplication by $e^{i \vartheta}$ for $\vartheta \in[0,2 \pi]$ and the complex conjugation. Therefore, Eq. (7.2) is equivalent to

$$
\left\{\begin{aligned}
u^{\prime}+\lambda_{1} u & =2\left(u^{2}+v^{2}\right) v \\
-v^{\prime}+\lambda_{1} v & =2\left(u^{2}+v^{2}\right) u
\end{aligned}\right.
$$

where $u=u_{1}=u_{2}, v=v_{1}=-v_{2}: \mathbb{R} / 2 \pi \ell \mathbb{Z} \rightarrow(0, \infty)$. Evidently, Eq. (7.3) has an "obvious" constant solution $u=v=\frac{\sqrt{\lambda_{1}}}{2}$ for all $\ell>0$.

From now on, we are intend to look for non-constant periodic solutions $u, v$ for Eq. (7.3). Setting $f=2 u^{2}+2 v^{2}$ and $g=2 u^{2}-2 v^{2}$, we have $u v=\frac{\sqrt{f^{2}-g^{2}}}{4}$ and Eq. (7.3) becomes

$$
\left\{\begin{array}{l}
g=-\frac{1}{2 \lambda_{1}} f^{\prime} \\
2 g g^{\prime}-2 f f^{\prime}=-\frac{2 f}{\lambda_{1}} f^{\prime} \sqrt{f^{2}-g^{2}} .
\end{array}\right.
$$


After multiplication by $\frac{1}{2}\left(f^{2}-g^{2}\right)^{-\frac{1}{2}}$ in the second equation, we have

$$
\frac{d}{d t}\left(\sqrt{f^{2}-g^{2}}\right)=\frac{d}{d t}\left(\frac{1}{2 \lambda_{1}} f^{2}\right) .
$$

Thus, for any solutions $f$ and $g$, there exists a constant $K$ such that $\sqrt{f^{2}-g^{2}}=\frac{1}{2 \lambda_{1}} f^{2}+K$, that is,

$$
g^{2}=f^{2}-\left(\frac{1}{2 \lambda_{1}} f^{2}+K\right)^{2} \text { and } \frac{1}{2 \lambda_{1}} f^{2}+K \geq 0 .
$$

For $K \in \mathbb{R}$, let us denote

$$
F_{K}(s)=s^{2}-\left(\frac{1}{2 \lambda_{1}} s^{2}+K\right)^{2} \text { for } s \geq 0 .
$$

Remark that, due to the geometric meaning of Eq. (7.1), the function $f$ defines a conformal metric $\tilde{g}=f^{2} g$ on $\ell S^{1} \times S^{1}$. And this, together with the first equation in (7.4), implies $F_{K}$ should vanish twice on at some points $s_{0}, s_{1}>0$. Thus, the condition on $K$ is particularly restrictive. In fact, the only possible range is $K \in\left(0, \frac{\lambda_{1}}{2}\right]$. And, if $K=\frac{\lambda_{1}}{2}$, we have $f \equiv \lambda_{1}$ and $g \equiv 0$ (which correspond exactly the constant solution $u=v=\frac{\sqrt{\lambda_{1}}}{2}$ ).

Let $K \in\left(0, \frac{\lambda_{1}}{2}\right)$, and take $0<s_{0}<s_{1}$ be the points such that $F_{K}$ vanishes. Then the function $F_{K}$ is positive on the interval $\left(s_{0}, s_{1}\right)$. And Eq. (7.5) is now equivalent to

$$
\frac{d f}{2 \lambda_{1} \sqrt{F_{K}(f)}}= \pm d t
$$

that is $\eta_{K}(f)= \pm t+c$, where

$$
\eta_{K}(f)=\int_{s_{0}}^{f} \frac{d s}{2 \lambda_{1} \sqrt{F_{K}(s)}} .
$$

Of course, $\eta_{K}$ is defined on the interval $\left(s_{0}, s_{1}\right)$. By noting that $s_{0}$ and $s_{1}$ are simple roots of $F_{K}$, we have $\eta_{K}$ is well-defined. Moreover, we have $\eta_{K}^{\prime}(s)=\frac{1}{2 \lambda_{1} \sqrt{F_{K}(s)}}>0$ and $\eta_{K}^{\prime}(s) \rightarrow+\infty$ as $s \rightarrow s_{0}$ or $s_{1}$. Hence $\eta_{K}$ has an inverse $\eta_{K}^{-1}$ which increases from $s_{0}$ to $s_{1}$ on the interval $\left[0, \eta_{K}\left(s_{1}\right)\right]$. And solutions to (7.5) can be given by $f(t)=\eta_{K}^{-1}( \pm t+c)$ for $c \in \mathbb{R}$.

Setting

$$
f_{K}(t)= \begin{cases}\eta_{K}^{-1}(t) & t \in\left[0, \eta_{K}\left(s_{1}\right)\right], \\ \eta_{K}^{-1}(-t) & t \in\left[-\eta_{K}\left(s_{1}\right), 0\right],\end{cases}
$$

it follows that the positive periodic solutions of Eq. (7.4) can be characterized by $f_{K}(t+c)$ which is a $2 \eta_{K}\left(s_{1}\right)$-periodic function. We are looking for solutions having period $2 \pi \ell$, that is, functions whose smallest positive period is of the form $2 \pi \ell / k$ for some $k \in \mathbb{N}$. Thus the nonconstant solutions of our problem are functions $f_{K, c}: t \mapsto f_{K}(t+c)$ for which there exists $k \in \mathbb{N}$ such that

$$
\eta_{K}\left(s_{1}\right)=\frac{\pi \ell}{k} .
$$

Lemma 7.1. $\eta_{K}\left(s_{1}\right)$ decreases with respect to the factor $K \in\left(0, \frac{\lambda_{1}}{2}\right)$. Particularly,

$$
\lim _{K \rightarrow 0} \eta_{K}\left(s_{1}\right) \rightarrow+\infty \quad \text { and } \quad \lim _{K \rightarrow \frac{\lambda_{1}}{2}} \eta_{K}\left(s_{1}\right)=\frac{\pi}{2 \lambda_{1}} .
$$


Proof. To begin with, let us rewrite $F_{K}$ in its factorization

$$
F_{K}(s)=\frac{1}{2 \lambda_{1}}\left(s-s_{0}\right)\left(s_{1}-s\right)\left(s+\frac{1}{2 \lambda_{1}} s^{2}+K\right) .
$$

in which we have the explicit formulation

$$
s_{0}=\lambda_{1}-\sqrt{\lambda_{1}^{2}-2 \lambda_{1} K} \quad \text { and } \quad s_{1}=\lambda_{1}+\sqrt{\lambda_{1}^{2}-2 \lambda_{1} K} .
$$

Then, we get

$$
\eta_{K}\left(s_{1}\right)=\int_{s_{0}}^{s_{1}} \frac{d s}{\sqrt{\left(s-s_{0}\right)\left(s_{1}-s\right)\left(s^{2}+2 \lambda_{1} s+2 \lambda_{1} K\right)}} .
$$

Consider the change of variable $s=s_{t}=s_{0}+\left(s_{1}-s_{0}\right) t, t \in[0,1]$, we obtain

$$
\eta_{K}\left(s_{1}\right)=\int_{0}^{1} \frac{d t}{\sqrt{t(1-t)\left(s_{t}^{2}+2 \lambda_{1} s_{t}+2 \lambda_{1} K\right)}} .
$$

For each $(t, K) \in(0,1) \times\left(0, \frac{\lambda_{1}}{2}\right)$, let's denote

$$
H(t, K)=\frac{1}{\sqrt{t(1-t)\left(s_{t}^{2}+2 \lambda_{1} s_{t}+2 \lambda_{1} K\right)}}
$$

Notice $s_{t}=\lambda_{1}-\sqrt{\lambda_{1}^{2}-2 \lambda_{1} K}(1-2 t)$, it follows from a straightforward calculation that

$$
\frac{\partial}{\partial K} H(t, K)=-H(t, K)^{3} \cdot t(1-t) \cdot\left[\left(s_{t}+\lambda_{1}\right) \frac{\partial s_{t}}{\partial K}+\lambda_{1}\right],
$$

where

$$
\left(s_{t}+\lambda_{1}\right) \frac{\partial s_{t}}{\partial K}=2 \lambda_{1}^{2}\left(\lambda_{1}^{2}-2 \lambda_{1} K\right)^{-\frac{1}{2}}(1-2 t)-\lambda_{1}(1-2 t)^{2} .
$$

Particularly, $\frac{\partial}{\partial K} H(t, K)$ has the following simplified formulation

$$
\frac{\partial}{\partial K} H(t, K)=\lambda_{1}\left(\lambda_{1}-2 \lambda_{1} K\right)^{-1} \cdot L(t, K) \cdot H(t, K)-\lambda_{1} H(t, K)^{3} \cdot t(1-t)
$$

with

$$
L(t, K)=\frac{\left(\lambda_{1}-\sqrt{\lambda_{1}^{2}-2 \lambda_{1} K}(1-2 t)\right)^{2}-\lambda_{1}^{2}}{\left(2 \lambda_{1}-\sqrt{\lambda_{1}^{2}-2 \lambda_{1} K}(1-2 t)\right)^{2}-\lambda_{1}^{2}+2 \lambda_{1} K} .
$$

Thus, we see easily that the map $t \mapsto \frac{\partial}{\partial K} H(t, K)$ is in $L^{1}(0,1)$ for all $K \in\left(0, \frac{\lambda_{1}}{2}\right)$.

Since $t \in(0,1)$, one checks that $L\left(\frac{1}{2}, K\right) \equiv 0$,

$$
L(t, K)<0 \text { if } 0 \leq t<\frac{1}{2} \text { and } L(t, K)>0 \text { if } \frac{1}{2}<t \leq 1 .
$$

Moreover, by an elementary computation, we can find

$$
-L(t, K)>L(1-t, K) \text { for all } t \in\left(0, \frac{1}{2}\right) .
$$


Notice that the values of the function $t \mapsto s_{t}^{2}+2 \lambda_{1} s_{t}+2 \lambda_{1} K$ for $t \in\left[0, \frac{1}{2}\right)$ is strictly smaller than that for $t \in\left(\frac{1}{2}, 1\right]$. Then, we can substitute (7.8) into (7.7) to get

$$
\frac{d}{d K} \eta_{K}\left(s_{1}\right)<\lambda_{1}\left(\lambda_{1}-2 \lambda_{1} K\right)^{-1}\left(\int_{0}^{\frac{1}{2}} L(t, K) \cdot H(t, K) d t+\int_{\frac{1}{2}}^{1} L(t, K) \cdot H(t, K) d t\right)<0
$$

which shows $\eta_{K}\left(s_{1}\right)$ is decreasing with respect to $K$.

In order to calculate the limits, let us mention that, as $K \rightarrow 0$, we have $s=s_{0}+\left(s_{1}-s_{0}\right) t \rightarrow$ $2 \lambda_{1} t$ for $t \in[0,1]$. Hence, for arbitrary $\delta>0$, it follows from Fatou's lemma that

$$
\begin{aligned}
\lim _{K \rightarrow 0} \eta_{K}\left(s_{1}\right) & \geq \lim _{K \rightarrow 0} \int_{\delta}^{\frac{1}{2}} \frac{d t}{\sqrt{t(1-t)\left(s^{2}+2 \lambda_{1} s+2 \lambda_{1} K\right)}} \\
& \geq \frac{1}{2 \lambda_{1}} \int_{\delta}^{\frac{1}{2}} \frac{d t}{t \sqrt{1-t^{2}}}>\frac{1}{2 \lambda_{1}}\left(\ln \frac{1}{2}-\ln \delta\right) .
\end{aligned}
$$

And thus, by taking $\delta \rightarrow 0$, we have $\lim _{K \rightarrow 0} \eta_{K}\left(s_{1}\right)=+\infty$.

For $K \rightarrow \frac{\lambda_{1}}{2}$, we shall use the fact $s_{0}, s_{1} \rightarrow \lambda$ to obtain

$$
\lim _{K \rightarrow \frac{\lambda_{1}}{2}} \eta_{K}\left(s_{1}\right)=\frac{1}{2 \lambda_{1}} \int_{0}^{1} \frac{d t}{\sqrt{t(1-t)}}=\frac{\pi}{2 \lambda_{1}}
$$

which completes the whole proof.

Recall that we are looking for the existence of $2 \eta_{K}\left(s_{1}\right)$-periodic solutions of Eq. (7.4) satisfying (7.6), then Lemma 7.1 implies

(1) For every $\ell>0$, Eq. (7.4) has the constant solution $f_{0} \equiv \lambda_{1}$ and $g_{0} \equiv 0$ which gives the constant solution $\psi_{1}=\frac{\sqrt{\lambda_{1}}}{2}+i \frac{\sqrt{\lambda_{1}}}{2}$ and $\psi_{2}=\frac{\sqrt{\lambda_{1}}}{2}-i \frac{\sqrt{\lambda_{1}}}{2}$ to Eq. (7.2). Such a solution satisfies

$$
\operatorname{Vol}\left(N, f_{0}^{2} g\right)=\int_{N=\ell S^{1} \times S^{1}} f_{0}^{2} d t d \tau=4 \pi^{2} \lambda_{1}^{2} \ell .
$$

And, for $\ell \leq \frac{1}{2 \lambda_{1}}$, this is the only solution of Eq. (7.4).

(2) Let $d \in \mathbb{N}$ with $\frac{d}{2 \lambda_{1}}<\ell \leq \frac{d+1}{2 \lambda_{1}}$. Then for any $k=1,2, \ldots, d$, we have $\frac{\pi \ell}{k} \geq \frac{\pi \ell}{d}>\frac{\pi}{2 \lambda_{1}}$ and there exists $K=K(\ell / k) \in\left(0, \frac{\lambda_{1}}{2}\right)$ such that $\eta_{K}\left(s_{1}\right)=\frac{\pi \ell}{k}$. And the solution $f_{k}$ of Eq. (7.4) corresponding to $K$ satisfies

$$
\operatorname{Vol}\left(N, f_{k}^{2} g\right)=\int_{\ell S^{1} \times S^{1}} f_{k}^{2} d t d \tau=\frac{2 k \pi}{\lambda_{1}} \int_{s_{0}}^{s_{1}} \frac{s^{2}}{\sqrt{F_{K}(s)}} d s .
$$

Lemma 7.2. For any $\ell>\frac{1}{2 \lambda_{1}}$, we have $\operatorname{Vol}\left(N, f_{1}^{2} g\right)<\min \left\{\operatorname{Vol}\left(N, f_{0}^{2} g\right), 8 \lambda_{1} \pi\right\}$.

Proof. Since $f_{1}$ has only one period on $\ell S^{1}$, by Lemma 7.1, we can fix $K=K(\ell)>0$ such that $\eta_{K}\left(s_{1}\right)=\pi \ell$. Following from (7.9) and (7.10), we have to show that

$$
\frac{1}{2 \lambda_{1}} \int_{s_{0}}^{s_{1}} \frac{s^{2}}{\sqrt{F_{K}(s)}} d s<\pi \lambda_{1}^{2} \ell .
$$


Similar to the calculations in Lemma 7.1, let us consider the change of variable $s=s_{t}=$ $s_{0}+\left(s_{1}-s_{0}\right) t$ for $t \in[0,1]$. Then we have

$$
\begin{aligned}
\pi \lambda_{1}^{2} \ell-\frac{1}{2 \lambda_{1}} \int_{s_{0}}^{s_{1}} \frac{s^{2}}{\sqrt{F_{K}(s)}} d s & =\int_{0}^{1} \frac{\lambda_{1}^{2}-s_{t}^{2}}{\sqrt{t(1-t)\left(s_{t}^{2}+2 \lambda_{1} s_{t}+2 \lambda_{1} K\right)}} d t \\
& =\sqrt{\lambda_{1}^{2}-2 \lambda_{1} K} \int_{0}^{1} \frac{(1-2 t) \cdot B(t)}{\sqrt{t(1-t)}} d t
\end{aligned}
$$

where

$$
B(t)=\frac{2 \lambda_{1}-\sqrt{\lambda_{1}^{2}-2 \lambda_{1} K}(1-2 t)}{\sqrt{\left(s_{t}^{2}+2 \lambda_{1} s_{t}+2 \lambda_{1} K\right)}} .
$$

Clearly, $B(t)>0$ for all $t \in(0,1)$. Furthermore, it follows immediately from the computations

$$
\frac{\left(s_{t}^{2}+2 \lambda_{1} s_{t}+2 \lambda_{1} K\right)^{\frac{3}{2}}}{2 \sqrt{\lambda_{1}^{2}-2 \lambda_{1} K}} B^{\prime}(t)=\left(s_{t}^{2}+2 \lambda_{1} s_{t}+2 \lambda_{1} K\right)-\left(2 \lambda_{1}-\sqrt{\lambda_{1}^{2}-2 \lambda_{1} K}(1-2 t)\right)^{2}
$$

and

$$
s_{t}^{2}+2 \lambda_{1} s_{t}+2 \lambda_{1} K=\left(2 \lambda_{1}-\sqrt{\lambda_{1}^{2}-2 \lambda_{1} K}(1-2 t)\right)^{2}-\lambda_{1}^{2}+2 \lambda_{1} K
$$

that $B^{\prime}(t)<0$. Therefore, we can see from (7.12) that the total integral is positive and this implies (7.11). And thus,

$$
\operatorname{Vol}\left(N, f_{1}^{2} g\right)<4 \pi^{2} \lambda_{1}^{2} \ell=\operatorname{Vol}\left(N, f_{0}^{2} g\right)
$$

To give another upper bound for $\operatorname{Vol}\left(N, f_{1}^{2} g\right)$, let us first set $\delta=\sqrt{\lambda_{1}^{2}-2 \lambda_{1} K} \in\left(0, \lambda_{1}\right)$. Then, for the change of variable $s=s_{t}=\lambda_{1}-\delta(1-2 t)$ for $t \in[0,1]$, we have

$$
\frac{1}{2 \lambda_{1}} \int_{s_{0}}^{s_{1}} \frac{s^{2}}{\sqrt{F_{K}(s)}} d s=\int_{0}^{1} \frac{s_{t}^{2}}{\sqrt{t(1-t)\left(s_{t}^{2}+2 \lambda_{1} s_{t}+2 \lambda_{1} K\right)}} d t<\int_{0}^{1} \frac{Z(t, \delta)}{\sqrt{t(1-t)}} d t
$$

where

$$
Z(t, \delta)=\frac{s_{t}^{2}}{\sqrt{s_{t}^{2}+2 \lambda_{1} s_{t}}}
$$

Notice that, for $x>0$,

$$
\frac{d}{d x}\left(\frac{x^{2}}{\sqrt{x^{2}+2 \lambda_{1} x}}\right)>0, \quad \frac{d^{2}}{d x^{2}}\left(\frac{x^{2}}{\sqrt{x^{2}+2 \lambda_{1} x}}\right)>0 .
$$

And, for $t \in(0,1)$,

$$
\frac{\partial}{\partial \delta} Z(t, \delta)=\frac{d}{d s_{t}}\left(\frac{s_{t}^{2}}{\sqrt{s_{t}^{2}+2 \lambda_{1} s_{t}}}\right) \frac{d s_{t}}{d \delta}=(2 t-1) \frac{d}{d s_{t}}\left(\frac{s_{t}^{2}}{\sqrt{s_{t}^{2}+2 \lambda_{1} s_{t}}}\right) .
$$

Hence we have

$$
\frac{d}{d \delta} \int_{0}^{1} \frac{Z(t, \delta)}{\sqrt{t(1-t)}} d t=\int_{0}^{1} \frac{\frac{\partial}{\partial \delta} Z(t, \delta)}{\sqrt{t(1-t)}} d t>0
$$


that is, the integral $\int_{0}^{1} \frac{Z(t, \delta)}{\sqrt{t(1-t)}} d t$ is strictly increasing with respect to $\delta$. At the same time, one calculates easily that

$$
\lim _{\delta \rightarrow \lambda_{1}} \int_{0}^{1} \frac{Z(t, \delta)}{\sqrt{t(1-t)}} d t=\int_{0}^{1} \frac{2 \lambda_{1} t^{2}}{\sqrt{t^{2}\left(1-t^{2}\right)}} d t=2 \lambda_{1}
$$

Therefore, we conclude

$$
\operatorname{Vol}\left(N, f_{1}^{2} g\right)=\frac{2 \pi}{\lambda_{1}} \int_{s_{0}}^{s_{1}} \frac{s^{2}}{\sqrt{F_{K}(s)}} d s<4 \pi \int_{0}^{1} \frac{Z(t, \delta)}{\sqrt{t(1-t)}} d t<8 \lambda_{1} \pi
$$

and, together with (7.13), we complete the proof.

Theorem 7.3. Let $\lambda>0$ denote a positive eigenvalue of the Dirac operator on the second circle in $N=\ell S^{1} \times S^{1}$, then the following facts valid

(1) For every $\ell>0$, the Spinorial Yamabe equation

$$
D_{g} \phi=|\phi|^{2} \phi \quad \text { on } N=\ell S^{1} \times S^{1}
$$

has a constant length solution

$$
\phi_{0}=e^{-i(\lambda \tau+\vartheta)}\left(\begin{array}{c}
\frac{\sqrt{\lambda}}{2}+i \frac{\sqrt{\lambda}}{2} \\
\frac{\sqrt{\lambda}}{2}-i \frac{\sqrt{\lambda}}{2}
\end{array}\right) \in \mathbb{C}^{2}
$$

for any $\vartheta \in[0,2 \pi]$ such that

$$
\operatorname{Vol}\left(N,\left|\phi_{0}\right|^{4} g\right)=4 \pi^{2} \lambda^{2} \ell
$$

And, for $\ell \leq \frac{1}{2 \lambda}$, this is the only solution of the form $\phi=\psi e^{-i \lambda \tau} \in \mathbb{S}(N)$ to Eq. (7.14).

(2) Let $\ell>\frac{1}{2 \lambda}$ and $d \in \mathbb{N}$ with $\frac{d}{2 \lambda}<\ell \leq \frac{d+1}{2 \lambda}$, Eq. (7.14) has $d+1$ inequivalent solutions. Particularly, these solutions are given by the constant length solution and $k$ periods of a solution $\phi_{\ell, k}$ on $N$ with fundamental period $\frac{2 \pi \ell}{k}$ for $k=1,2, \ldots, d$.

(3) Let $\ell>\frac{1}{2 \lambda}$ and $\phi_{\ell, 1}$ denote the $2 \pi \ell$-periodic solution of Eq. (7.14), then

$$
\operatorname{Vol}\left(N,\left|\phi_{\ell, 1}\right|^{4} g\right)<\min \left\{\operatorname{Vol}\left(N,\left|\phi_{0}\right|^{4} g\right), 8 \pi \lambda\right\}
$$

and

$$
\lim _{\ell \rightarrow \infty} \operatorname{Vol}\left(N,\left|\phi_{\ell, 1}\right|^{4} g\right)=8 \pi \lambda
$$

(4) Let $\sigma_{N}^{*}$ denote the nontrivial spin structure on $N=\ell S^{1} \times S^{1}$ such that $\lambda=\frac{1}{2}$ is the first positive eigenvalue of the Dirac operator on the second circle, then

$$
\lambda_{\min }^{+}\left(N, g, \sigma_{N}^{*}\right) \leq \operatorname{Vol}\left(N,\left|\phi_{\ell, 1}\right|^{4} g\right)^{\frac{1}{2}}<2 \sqrt{\pi}
$$

for all $\ell>0$. 


\section{References}

[1] N. Ackermann, A nonlinear superposition principle and multibump solution of periodic Schrödinger equations, J. Funct. Anal. 234 (2006), 423-443.

[2] R. Adams, Sobolev Spaces, Academic Press, New York, (1975).

[3] H. Ammann, Saddle points and multiple solutions of differential equations, Math. Z. 169 (1979), no. 2, 127-166.

[4] B. Ammann, A variational problem in conformal spin geometry, Habilitationsschift, Universität Hamburg, (2003).

[5] B. Ammann, A spin-conformal lower bound of the first positive Dirac eigenvalue, Differ. Geom. Appl. 18 (2003), 21-32.

[6] B. Ammann, The smallest Dirac eigenvalue in a spin-conformal class and cmc immersions, Comm. Anal. Geom. 17 (2009), no. 3, 429-479.

[7] B. Ammann, M. Dahl, A. Hermann, E. Humbert, Mass endomorphism, surgery and perturbations, Ann. Inst. Fourier. 64 (2014), no. 2, 467-487.

[8] B. Ammann, J.-F. Grossjean, E. Humbert, B. Morel, A spinorial analogue of Aubin's inequality, Math. Z. 260 (2008), 127-151.

[9] B. Ammann, E. Humbert, M. Ould. Ahmedou, An obstruction for the mean curvature of a conformal immersion $S^{n} \rightarrow \mathbb{R}^{n+1}$, Proc. Amer. Math. Soc. 135 (2007), no. 2, 489-493.

[10] B. Ammann, E. Humbert, B. Morel, Mass endomorphism and spinorial Yamabe type problems on conformally flat manifolds, Comm. Anal. Geom. 14 (2006), no. 1, 163-182.

[11] M.T. Anderson, On uniqueness and differentiability in the space of Yamabe metrics, Commun. Contemp. Math. 7 (3) (2005) 299-310.

[12] T. Aubin, Équations différentielles non linéaires et problème de Yamabe concernant la courbure scalaire, J. Math. Pures Appl. 55 (1976), 269-296.

[13] C. Bär, Lower eigenvalue estimates for Dirac operators, Math. Ann. 293 (1992), 39-46.

[14] C. Bär, Extrinsic bounds for eigenvalues of the Dirac operator, Ann. Global Anal. Geom. 16 (1998), no. 6, 573-596.

[15] T. Bartsch, Y.H. Ding, Deformation theorems on non-metrizable vector spaces and applications to critical point theory, Math. Nachrichten, 279(12), (2006): 1267-1288.

[16] V. Benci, P.H. Rabinowitz, Critical point theorems for indefinite functionals, Invent. Math. 52 (1979), no. 3, 241-273. 
[17] R. G. Bettiol and P. Piccione. Multiplicity of solutions to the Yamabe problem on collapsing Riemannian submersions. Pacific J. Math., 266 (2013), 1-21.

[18] R. G. Bettiol and P. Piccione. Infinitely many solutions to the Yamabe problem on noncompact manifolds. Ann. Inst. Fourier (Grenoble), 68 (2018), 589-609.

[19] R. G. Bettiol, P. Piccione, and B. Santoro. Bifurcation of periodic solutions to the singular Yamabe problem on spheres. J. Differential Geom., 103 (2016), 191-205.

[20] R. G. Bettiol, P. Piccione, and Y. Sire. Nonuniqueness of conformal metrics with constant Q-curvature. To appear in IMRN.

[21] J.-P. Bourguignon, P. Gauduchon, Spineurs, opérateurs de Dirac et variations de métriques, Comm. Math. Phys. 144 (1992), no. 3, 581-599.

[22] B. Buffoni, L. Jeanjean, C.A. Stuart, Existence of a non-trivial solution to a strongly indefinite semilinear equation, Proc. Amer. Math. Soc., 119 (1993), 179-186.

[23] T. Friedrich, On the spinor representation of surfaces in Euclidean 3-space, J. Geom. Phys. 28 (1998), no. 1-2, 143-157.

[24] T. Friedrich, Dirac Operators in Riemannian Geometry, Grad. Stud. Math., vol 25, Amer. Math. Soc., Providence (2000).

[25] N. Ginoux, The Dirac Spectrum, Lecture Notes in Mathematics, vol. 1976. Springer, Berlin (2009).

[26] E. Hebey, M. Vaugon, Meilleures constantes dans le théorème d'inclusion de Sobolev et multiplicité pour les problèmes de Nirenberg et Yamabe, Indiana Univ. Math. J. 41 (2) (1992) 377-407.

[27] O. Hijazi, A conformal lower bound for the smallest eigenvalue of the Dirac operator and Killing spinors, Comm. Math. Phys. 104 (1986), 151-162.

[28] O. Hijazi, Spectral properties of the Dirac operator and geometrical structures, in Geometric Methods for Quantum Field Theory. Proceedings of the Summer School, eds. H. Ocampo et al., Villa de Leyva, Colombia, July 12-30, 1999, World Scientific, Singapore, 2001, 116-169.

[29] N. Hitchin, Harmonic spinors, Adv. Math. 14 (1974), 1-55.

[30] H. Hofer, On strongly indefinite functionals with applications, Trans. Amer. Math. Soc. 275 (1983), no. 1, 185-214.

[31] O. Kobayashi, Scalar curvature of a metric with unit volume, Math. Ann. 279 (2) (1987) 253-265. 
[32] W. Kryszewski, A. Szulkin, Generalized linking theorem with an application to semilinear Schrödinger equation, Adv. Diff. Equ., 3, (1998): 441-472.

[33] R. Kusner, N. Schmitt, Representation of surfaces in space, arXiv:dg-ga/9610005, (1996).

[34] H.B. Lawson, M.L. Michelson, Spin Geometry, Princeton University Press (1989).

[35] P.L. Lions, The concentration-compactness principle in the calculus of variations: The locally compact case, Part II, AIP Anal. non linéaire 1 (1984), 223-283.

[36] J. Lott, Eigenvalue bounds for the Dirac operator, Pac. J. Math. 125 (1986), 117-126.

[37] R. Miguel, T. Hugo, Solutions with multiple spike patterns for an elliptic system. Calc. Var. Partial Differential Equations (2008) 31(1), 1-25.

[38] R. Miguel, J.F. Yang, Spike-layered solutions for an elliptic system with Neumann boundary conditions, Trans. Amer. Math. Soc. 357 (2005) 3265-3284.

[39] J. Petean, Metrics of constant scalar curvature conformal to Riemannian products, Proc. Amer. Math. Soc. 138 (8) (2010) 2897-2905.

[40] M. Reed, B. Simon, Methods of Mathematical Physics, Vols. I-IV, Academic Press, 1978.

[41] R. Schoen, Conformal deformation of a Riemannian metric to constant scalar curvature, J. Differential Geometry 20 (1984), 479-495.

[42] R. Schoen, Variational theory for the total scalar curvature functional for Riemannian metrics and related topics, in: Topics in Calculus of Variations, in: Lecture Notes in Math., vol. 1365, 1989, pp. 120-154.

[43] A. Szulkin, T. Weth, The method of Nehari manifold. Handbook of nonconvex analysis and applications, 597-632, Int. Press, Somerville, MA, 2010.

[44] N. Trudinger, Remarks concerning the conformal deformation of Riemannian structures on compact manifolds, Ann. Scuola Norm. Sup. Pisa 22 (1968), 265-274.

[45] H. Yamabe, On the deformation of Riemannian structures on compact manifolds, Osaka Math. J. 12 (1960) 21-37.

YANNICK SIRE

Department of Mathematics, Johns Hopkins University, 3400 N. Charles Street, Baltimore, Maryland 21218

sire@math.jhu.edu

TIAN XU

Center for Applied Mathematics, Tianjin University, 300072, TIANJin, CHINA

xutian@amss.ac.cn 